\title{
Compressive Sensing Super Resolution from Multiple Observations with Application to Passive Millimeter Wave Images
}

\author{
Wael AlSaafin ${ }^{\text {ta }}$, Salvador Villena ${ }^{\mathrm{b}}$, Miguel Vega $^{\mathrm{b}}$, Rafael Molina $^{\mathrm{a}}$, \\ Aggelos K. Katsaggelos ${ }^{\mathrm{c}}$ \\ ${ }^{a}$ Dept. of Computer Science and Artificial Intelligence, University of Granada, Spain. \\ ${ }^{b}$ Dept. of Languages and Information Systems, University of Granada, Spain. \\ ${ }^{c}$ Dept. of Electrical Engineering and Computer Science, Northwestern University, USA
}

\begin{abstract}
In this work we propose a novel framework to obtain high resolution images from compressed sensing imaging systems capturing multiple low resolution images of the same scene. The proposed approach of Compressed Sensing Super Resolution (CSSR), combines existing compressed sensing reconstruction algorithms with a low-resolution to high-resolution approach based on the use of a super Gaussian regularization term. The reconstruction alternates between compressed sensing reconstruction and super resolution reconstruction, including registration parameter estimation. The image estimation subproblem is solved using majorization-minimization while the compressed sensing reconstruction becomes an $l_{1}$-minimization subject to a quadratic constraint. The performed experiments on grayscale and synthetically compressed real millimeter wave images, demostrate the capability of the proposed framework to provide very good quality super resolved images from multiple low resolution compressed acquisitions.
\end{abstract}

Keywords: Super resolution, compressed sensing, image reconstruction, passive millimeter wave images.

\section{Introduction}

Compressed Sensing (CS) theory offers a framework to simultaneously sense and compress signals. It establishes that a sparsely representable image/signal can be recovered from a highly incomplete set of measurements [1 3 .

The design of CS image/video cameras (see [4] 8]) has fostered the application of typical image processing tasks to CS observed images. CS has been

\footnotetext{
* Corresponding author

Email addresses: waelsaafin@hotmail.com (Wael AlSaafin ${ }^{\star ै}$ ), svillena@ugr.es (Salvador Villena), mvega@ugr.es (Miguel Vega), rms@decsai.ugr.es (Rafael Molina), aggk@eecs.northwestern.edu (Aggelos K. Katsaggelos)
}

Preprint submitted to Elsevier

November 12, 2015

(C) 2015. This manuscript version is made available under the Elsevier user license http://www.elsevier.com/open-access/userlicense/1.0/ 
applied to areas like radar analysis, face recognition, biomedical imaging, and microscopy imaging techniques [2, 9, 10, among others. CS measurements have also been used to recover images observed through unknown blur [11, 12].

Super Resolution (SR) from a single image has also benefited from the introduction of CS theory. In [13, 14] learning based SR is used to estimate a High Resolution (HR) image from the CS observation of a downsampled remote sensing image. In [15] the downsampling is incorporated in the measurement matrix, the CS image is reconstructed in the wavelet domain and the signal is deconvolved in the Fourier domain.

The recovery of an HR image from a set of unregistered LR CS observations has been scarcely addressed in the literature. To the best of our knowledge, the only reported works treating this general SR problem are [16, 17. In these papers CS and LR to HR techniques are coupled, using a fast and simple registration method, which uses the reconstructed HR images instead of the LR ones [17]. A non robust prior model on the original image to be reconstructed was used in both papers.

This paper also deals with the reconstruction of an HR image from a group of LR CS observed images. The proposed method assumes that the HR image to be estimated is compressible and, consequently, its warped, blurred, and downsampled versions are also compressible (see [11, 12]). They can then be reconstructed from their CS observations. However, instead of first recovering the LR observations and then using LR to HR techniques we propose a combined framework where LR reconstructions and HR estimation are carried out simultaneously. This proposed method is based on a sound and well founded method to estimate registration parameters in LR to HR problems and the use of a new robust sparse promoting prior for the original image.

The proposed framework has been tested, in the experimental section, on CS grayscale and Passive Millimeter Wave (PMMW) images. Without using CS measurements, the improvement of Passive Millimeter Wave (PMMW) images to perform detection tasks has been addressed in [18 22, and the use of CS techniques to reduce the time needed to capture such images has been addressed in [5, 6, 23, 24. In [17, high resolution images were super resolved for the first time, from multiple CS observations of unregistered LR PMMW images. We believe that PMMW images represent an important application area where CS and LR to HR techniques can be combined to enhance the detection capabilities of current PMMWI systems.

Before going into details, the more frequently used notation in the paper is 
listed next

$$
\begin{aligned}
& \mathbf{y}_{q} \quad M \times 1 \text { compressed observation vector } q \in\{1, \cdots, Q\} \\
& \mathbf{\Phi} \quad M \times D \text { CS measurement matrix } \\
& \mathbf{z}_{q} \quad D \times 1 \text { the q-th LR image vector } \\
& \mathbf{r}_{q} \quad D \times 1 \text { CS observation noise vector } \\
& \text { A } \quad D \times N \text { down-sampling matrix } \\
& P \quad \text { zooming factor } \\
& \mathbf{H}_{q} \quad N \times N \text { blurring matrix } \\
& \mathbf{C}\left(\mathbf{s}_{q}\right) \quad N \times N \text { warping matrix formed by motion vector } \mathbf{s}_{q} \\
& \mathbf{s}_{q} \quad 3 \times 1 \text { motion vector (rotation } \theta_{q} \text {, horizontal } c_{q} \text {, and } \\
& \text { vertical } d_{q} \text { displacements) } \\
& \text { x } \quad N \times 1 \text { HR image vector } \\
& \mathbf{w}_{q} \quad D \times 1 \mathrm{HR} \text { to LR acquisition noise vector } \\
& \mathbf{B}_{q}\left(\mathbf{s}_{q}\right) \quad D \times N \text { LR acquisition model matrix } \\
& a_{q}\left(\mathbf{s}_{q}\right), b_{q}\left(\mathbf{s}_{q}\right) \quad N \times 1 \text { pixel difference vectors } \\
& \mathbf{D}_{a_{q}\left(\mathbf{s}_{q}\right)} \quad N \times N \text { diagonal matrix with } a_{q}\left(\mathbf{s}_{q}\right) \text { in the diagonal } \\
& \mathbf{D}_{b_{q}\left(\mathbf{s}_{q}\right)}: b_{q}\left(\mathbf{s}_{q}\right) \text { in the diagonal } \\
& \text { I the identity matrix }
\end{aligned}
$$

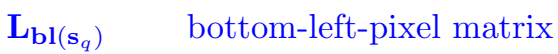

$$
\begin{aligned}
& \text { (br, tl, tr) : (bottom-right, top-left, top-right) } \\
& \mathbf{n}_{q} \quad M \times 1 \text { combined CS and LR acquisition noise vector } \\
& \text { W } \quad D \times D \text { transformation matrix } \\
& \mathbf{a}_{q} \quad D \times 1 \text { transformation coefficient vector } \\
& \alpha, \beta, \eta, \tau \quad \text { non-negative parameters } \\
& \mathbf{Q}(\mathbf{x}) \quad \text { regularization term } \\
& \boldsymbol{\omega}_{d}^{\mathbf{x}} \quad \text { filtered image applying } \mathbf{F}_{d} \text { on } \mathbf{x} \text { in } d \text {-direction } \\
& \boldsymbol{\lambda}_{q} \quad D \times 1 \text { Lagrangian multiplier vector }
\end{aligned}
$$

The rest of this paper is organized as follows. The problem modeling and its formulation as an optimization task are presented in Sections 2 and 3 . respectively. The estimation process is described in Section 4 . We demonstrate the effectiveness of the proposed method in the experimental section, Section 5 . Finally, conclusions are drawn in Section 6. 


\section{Modeling}

In this work we assume that we have access to a set of $Q$ CS LR observations of the form

$$
\mathbf{y}_{q}=\mathbf{\Phi} \mathbf{z}_{q}+\mathbf{r}_{q} \quad q=1, \ldots, Q
$$

where $\mathbf{y}_{q}$ is an $M \times 1$ vector representing compressed observations from the LR image $\mathbf{z}_{q}, \mathbf{\Phi}$ is a CS $M \times D$ measurement matrix, $\mathbf{z}_{q}$ is a column vector of size $D \times 1$ representing the $q$-th LR image and $\mathbf{r}_{q}$ represents the observation noise. We denote by $\mathrm{R}$ the compression ratio of the measurement system, that is $\mathrm{R}=M / D, R \leq 1$. The sensing matrix $\boldsymbol{\Phi}$ consists of either real or binary entries. The matrix used in our work is binary, since it is easier to be synthesized physically [6, 23, 24]. In both cases the rows/columns of $\boldsymbol{\Phi}$ are normalized to 1. We assume that the LR observations $\mathbf{z}_{q}$ are related to an HR image of size $\mathrm{N}$, denoted by the column vector $\mathbf{x}$ by

$$
\mathbf{z}_{q}=\mathbf{A} \mathbf{H}_{q} \mathbf{C}\left(\mathbf{s}_{q}\right) \mathbf{x}+\mathbf{w}_{q}=\mathbf{B}_{q}\left(\mathbf{s}_{q}\right) \mathbf{x}+\mathbf{w}_{q},
$$

where $\mathbf{A}$ is a $D \times N$ down-sampling matrix, $D \leq N$, which models the limited resolution of the acquisition system, when capturing the high resolution image, where $N=P^{2} D$ and $\mathrm{P} \geq 1$ is the zooming factor, in each dimension of the image. $\mathbf{H}_{q}$ is an $N \times N$ blurring matrix, modeling the action accompanying the imaging process. In this work, $\mathbf{H}_{q}$ is assumed to be known. $\mathbf{C}\left(\mathbf{s}_{q}\right)$ is the $N \times N$ warping matrix formed by motion vector $\mathbf{s}_{q}=\left[\theta_{q}, c_{q}, d_{q}\right]^{t}$, where $\theta_{q}$ is the rotation angle, and $c_{q}$ and $d_{q}$ are respectively the horizontal and vertical translations of the $q$-th LR image with respect to the reference frame. Finally, $\mathbf{w}_{q}$ models the noise associated to the HR to LR acquisition process. We write $\mathbf{B}_{q}\left(\mathbf{s}_{q}\right)=\mathbf{A} \mathbf{H}_{q} \mathbf{C}\left(\mathbf{s}_{q}\right)$ for simplicity.

As explained in [25], matrices $\mathbf{C}\left(\mathbf{s}_{q}\right)$ can be explicitly stated as follows. Let us denote the coordinates of the reference HR grid by $(u, v)$ and the coordinates of the $q^{\text {th }}$ warped HR grid, after applying $\mathbf{C}\left(\mathbf{s}_{q}\right)$ to $\mathbf{x}$, by $\left(u_{q}, v_{q}\right)$. Then it holds that

$$
\begin{aligned}
& u_{q}=u \cos \left(\theta_{q}\right)-v \sin \left(\theta_{q}\right)+c_{q} \\
& v_{q}=u \sin \left(\theta_{q}\right)+v \cos \left(\theta_{q}\right)+d_{q} .
\end{aligned}
$$

Let us denote the displacements between the grids by $\Delta\left(u_{q}, v_{q}\right)^{T}=(u, v)^{T}-$ $\left(u_{q}, v_{q}\right)^{T}$. The vector difference between the pixel at $\left(u_{q}, v_{q}\right)$ and the pixel at its top-left position in the reference HR grid is denoted by $\left(a_{q}\left(\mathbf{s}_{q}\right), b_{q}\left(\mathbf{s}_{q}\right)\right)^{T}$ (see Figure 1), that is,

$$
\begin{aligned}
& a_{q}\left(\mathbf{s}_{q}\right)=\Delta u_{q}-\text { floor }\left(\Delta u_{q}\right), \\
& b_{q}\left(\mathbf{s}_{q}\right)=\Delta v_{q}-\text { floor }\left(\Delta v_{q}\right) .
\end{aligned}
$$

Using bilinear interpolation, the warped image $\mathbf{C}\left(\mathbf{s}_{q}\right) \mathbf{x}$ can be approximated as

$$
\begin{aligned}
\mathbf{C}\left(\mathbf{s}_{q}\right) \mathbf{x} & \approx \mathbf{D}_{b_{q}\left(\mathbf{s}_{q}\right)}\left(\mathbf{I}-\mathbf{D}_{a_{q}\left(\mathbf{s}_{q}\right)}\right) \mathbf{L}_{\mathbf{b l}\left(\mathbf{s}_{q}\right)} \mathbf{x}+\left(\mathbf{I}-\mathbf{D}_{b_{q}\left(\mathbf{s}_{q}\right)}\right) \mathbf{D}_{a_{q}\left(\mathbf{s}_{q}\right)} \mathbf{L}_{\mathbf{t r}\left(\mathbf{s}_{q}\right)} \mathbf{x} \\
& +\left(\mathbf{I}-\mathbf{D}_{b_{q}\left(\mathbf{s}_{q}\right)}\right)\left(\mathbf{I}-\mathbf{D}_{a_{q}\left(\mathbf{s}_{q}\right)}\right) \mathbf{L}_{\mathbf{t l}\left(\mathbf{s}_{q}\right)} \mathbf{x}+\mathbf{D}_{b_{q}\left(\mathbf{s}_{q}\right)} \mathbf{D}_{a_{q}\left(\mathbf{s}_{q}\right)} \mathbf{L}_{\mathbf{b r}\left(\mathbf{s}_{q}\right)} \mathbf{x},
\end{aligned}
$$




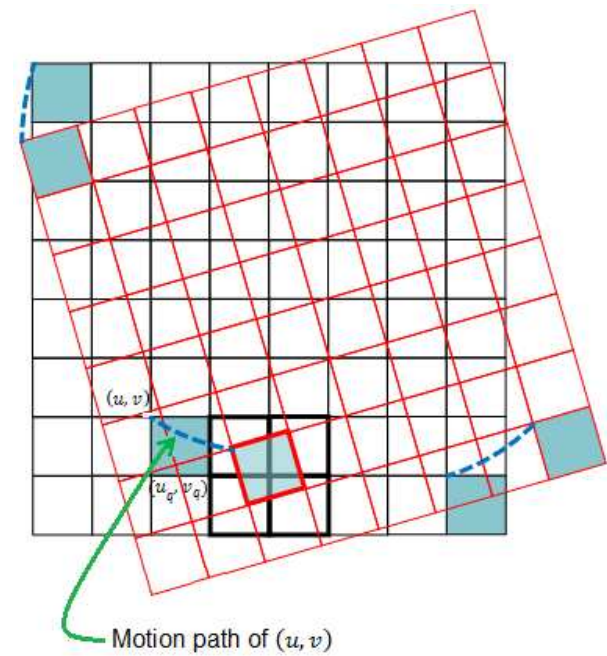

(a)

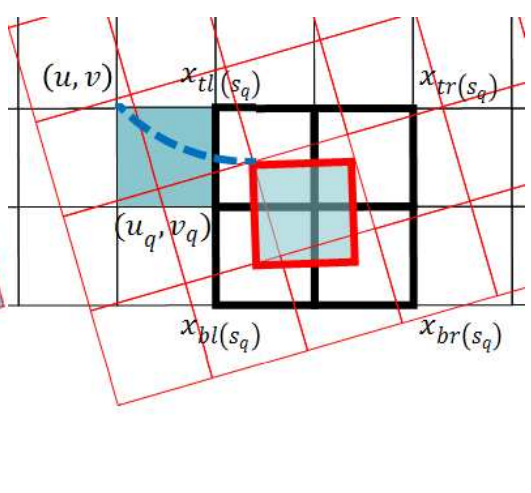

(b)

Figure 1: q-th HR grid calculation. (a) HR grid (in black) and the q-th image grid (in red), (b) Detailed view of (a), with the pixel notation used for the bilinear interpolation of grid element $\left(u_{q}, v_{q}\right)$

where $\mathbf{D}_{a_{q}\left(\mathbf{s}_{q}\right)}$ and $\mathbf{D}_{b_{q}\left(\mathbf{s}_{q}\right)}$ denote diagonal matrices with the vectors $a_{q}\left(\mathbf{s}_{q}\right)$ and $b_{q}\left(\mathbf{s}_{q}\right)$ in their diagonals, respectively. $\mathbf{I}$ is the identity matrix. Matrices $L_{\mathbf{z}}$ with $\mathbf{z} \in\left\{\mathbf{b l}\left(\mathbf{s}_{q}\right), \mathbf{b r}\left(\mathbf{s}_{q}\right), \operatorname{tl}\left(\mathbf{s}_{q}\right), \operatorname{tr}\left(\mathbf{s}_{q}\right)\right\}$ are constructed in such a way that the product $L_{\mathbf{z}} \mathbf{x}$ produces pixels at the bottom-left, bottom-right, top-left, and top-right, locations of $\left(u_{q}, v_{q}\right)$, respectively.

Using (1) and (2) we can write

$$
\mathbf{y}_{q}=\mathbf{\Phi} \mathbf{B}_{q}\left(\mathbf{s}_{q}\right) \mathbf{x}+\mathbf{n}_{q}, \text { for } q=1, \ldots, Q,
$$

where $\mathbf{n}_{q}$ represents the combined CS and LR acquisition noise and $\mathbf{x}$ is the HR image we want to estimate.

\section{Problem Formulation}

Since $\mathbf{z}_{q}$ in 2 represents translated and rotated LR versions of the original image $\mathbf{x}$ (which are assumed to be compressible in a transformed domain), we can estimate the original HR image by first recovering the LR images using CS techniques and then recover the HR image using standard super resolution techniques on the recovered low resolution images. To be precise, if we assume that the LR images are sparse in a transformed domain with $\mathbf{z}_{q}=\mathbf{W} \mathbf{a}_{q}$, where $\mathbf{W}$ is a sparse promoting transformation of size $D \times D$, we can recover them from the model in (1) by solving

$$
\hat{\mathbf{a}}_{q}=\arg \min _{\mathbf{a}_{q}} \frac{\eta}{2}\left\|\mathbf{\Phi} \mathbf{W} \mathbf{a}_{q}-\mathbf{y}_{q}\right\|^{2}+\tau\left\|\mathbf{a}_{q}\right\|_{1},
$$


where $\eta, \tau$ are regularization parameters, $\|\cdot\|$ is the Euclidean norm, and $\|\cdot\|_{1}$ the $\ell 1$ norm. Then defining $\hat{\mathbf{z}}_{q}=\mathbf{W} \hat{\mathbf{a}}_{q}$ and $\mathbf{s}=\left(\mathbf{s}_{1}, \ldots, \mathbf{s}_{Q}\right)$ and using the degradation model (2), we can estimate the original image by solving

$$
\hat{\mathbf{x}}, \hat{\mathbf{s}}=\arg \min _{\mathbf{x}, \mathbf{s}} \frac{\beta}{2} \sum_{q}\left\|\mathbf{B}_{q}\left(\mathbf{s}_{q}\right) \mathbf{x}-\hat{\mathbf{z}}_{q}\right\|^{2}+\alpha \mathbf{Q}(\mathbf{x}),
$$

where $\alpha$ and $\beta$ are non-negative parameters, and the remaining terms are described in details next. In [16, 17] the following regularization term, $\mathbf{Q}(\mathbf{x})$, was used for the image

$$
\mathbf{Q}(\mathbf{x})=\sum_{d \in \Delta} \sum_{i=1}^{N} \log \left(\left|\omega_{d}^{\mathbf{x}}(i)\right|\right),
$$

where $\omega_{d}^{\mathbf{x}}(i)$ is the $i$-th pixel of the filtered image, and

$$
\boldsymbol{\omega}_{d}^{\mathbf{x}}=\omega_{d}^{\mathbf{x}}(i) \mathbf{x},
$$

where $\mathbf{F}_{d}$ is a high-pass filter operator, and the index $d \in \Delta$ identifies one of the members of the used filter set. In this paper we have used a filter set with elements $\Delta=\{h, v, h v, v h, h h, v v\}$, where $h, v$ represent the first order horizontal and vertical difference filters, $h v$ and $v h$ represent first order differences along diagonals, and $h h$ and $v v$ the horizontal and vertical second order differences. The regularization term favors sparsity of the high-pass filtered images $\mathbf{F}_{d} \mathbf{x}$, and corresponds to the Super-Gaussian log prior used in blind deconvolution [26.

Since the log function can not be differentiated at zero, we consider in this work the following robust version of the log regularizer

$$
\log _{\epsilon}(|s|)= \begin{cases}\log (|s|) & \text { for }|s| \geq \epsilon \\ \frac{s^{2}}{2 \epsilon^{2}}-\left(\frac{1}{2}-\log (\epsilon)\right) & \text { for } 0 \leq|s| \leq \epsilon\end{cases}
$$

and replace $\mathbf{Q}(\mathbf{x})$ in 11 by

$$
\mathbf{Q}(\mathbf{x})=\sum_{d \in \Delta} \sum_{i=1}^{N} \log _{\epsilon}\left(\left|\omega_{d}^{\mathbf{x}}(i)\right|\right),
$$

where we have removed the dependency of $\mathbf{Q}(\cdot)$ on $\epsilon$ for simplicity.

We have two approaches to solve the CSSR problem: the sequential approach and the alternate approach (see [27]). The latter alternates between compressive sensing reconstruction, registration parameter estimation, and recovering of the HR image. The former approach estimates the unknowns sequentially, one after the other, as follows. Firstly, the LR images are reconstructed using (9), then motion parameters and the HR image are estimated using (10).

As we will show in the experimental section, combining the two optimization problems $(9,, 10$ ) above into a simultaneous one leads to improved performance, 
as this enables better exploitation of the compressibility of the LR observations using the additional information obtained from the estimated HR image. Hence, in the following, this alternate approach has been adopted. According to it, let $\mathbf{a}=\left(\mathbf{a}_{1}, \ldots, \mathbf{a}_{Q}\right)$ and define

$$
L(\mathbf{x}, \mathbf{a})=\frac{\eta}{2} \sum_{q=1}^{Q}\left\|\mathbf{\Phi} \mathbf{W} \mathbf{a}_{q}-\mathbf{y}_{q}\right\|^{2}+\tau \sum_{q=1}^{Q}\left\|\mathbf{a}_{q}\right\|_{1}+\alpha \mathbf{Q}(\mathbf{x}) .
$$

Then we approach the Compressed-Sensing Super Resolution (CSSR) problem by solving the following constrained optimization problem

$$
\begin{array}{ll}
\min & L(\mathbf{x}, \mathbf{a}) \\
\text { s.t. } & \mathbf{B}_{q}\left(\mathbf{s}_{q}\right) \mathbf{x}=\mathbf{W} \mathbf{a}_{q}, \text { for } q=1, \ldots, Q \text {. }
\end{array}
$$

This is the approach we will describe and use in the following section. Notice that in 15 we could have also introduced a regularizer on the motion vector $\mathbf{s}=\left(\mathbf{s}_{1}, \ldots, \mathbf{s}_{Q}\right)$. However, we have experimentally found that it is not necessary to use regularization on the motion vectors.

\section{A Super-Resolution from Compressed Sensing Algorithm}

The constrained optimization problem in 16 is converted into an unconstrained optimization one, using the Alternate Direction Method of Multipliers (ADMM) [28, 29]. We define the following augmented Lagrangian functional

$$
\begin{aligned}
L(\mathbf{x}, \mathbf{a}, \mathbf{s}, \boldsymbol{\lambda}) & =L(\mathbf{x}, \mathbf{a})+\sum_{q=1}^{Q} \boldsymbol{\lambda}_{q}^{t}\left(\mathbf{B}_{q}\left(\mathbf{s}_{q}\right) \mathbf{x}-\mathbf{W} \mathbf{a}_{q}\right) \\
& +\frac{\beta}{2} \sum_{q=1}^{Q}\left\|\mathbf{B}_{q}\left(\mathbf{s}_{q}\right) \mathbf{x}-\mathbf{W} \mathbf{a}_{q}\right\|^{2}
\end{aligned}
$$

where $L(\mathbf{x}, \mathbf{a})$ has been defined in 15 for $q=1, \ldots, Q, \boldsymbol{\lambda}_{q}$ are $D \times 1$ Lagrangian multiplier vectors with $\boldsymbol{\lambda}=\left(\boldsymbol{\lambda}_{1}, \ldots, \boldsymbol{\lambda}_{Q}\right)$, and $\beta$ is a non-negative parameter. The ADMM leads to the following sequence of iterative unconstrained problems,

$$
\begin{aligned}
& \mathbf{x}^{k+1}=\arg \min _{\mathbf{x}} L\left(\mathbf{x}, \mathbf{a}^{k}, \mathbf{s}^{k}, \boldsymbol{\lambda}^{k}\right) \\
& \mathbf{a}^{k+1}=\arg \min _{\mathbf{a}} L\left(\mathbf{x}^{k+1}, \mathbf{a}, \mathbf{s}^{k}, \boldsymbol{\lambda}^{k}\right) \\
& \mathbf{s}^{k+1}=\arg \min _{\mathbf{s}} L\left(\mathbf{x}^{k+1}, \mathbf{a}^{k+1}, \mathbf{s}, \boldsymbol{\lambda}^{k}\right) \\
& \boldsymbol{\lambda}_{q}^{k+1}=\boldsymbol{\lambda}_{q}^{k}-\beta\left[\mathbf{B}_{q}\left(\mathbf{s}_{q}^{k+1}\right) \mathbf{x}^{k+1}-\mathbf{W} \mathbf{a}_{q}^{k+1}\right], \quad q=1, \ldots, Q,
\end{aligned}
$$

where $k$ is the iteration number. Notice that according to the ADMM approach, $\mathbf{B}_{q}\left(\mathbf{s}_{q}\right)$ in 16 should not depend on the iteration index, as is the case here. 
However, we have not encountered any convergence issues with this iterative procedure.

Let us now describe the estimation process. The calculation of each $\boldsymbol{\lambda}_{q}^{k+1}$ is straightforward. The function $\rho_{\epsilon}(s)=\log _{\epsilon}(|s|)$ in $(14)$ is symmetric around 0 , and $\rho(\sqrt{s})$ is concave and increasing for $s \in[0, \infty)[26]$. So, it can be represented as (see [30])

$$
\rho_{\epsilon}(s)=\inf _{\xi>0} \frac{1}{2} \xi s^{2}-\rho_{\epsilon}^{*}\left(\frac{1}{2} \xi\right)
$$

where $\rho_{\epsilon}^{*}\left(\frac{1}{2} \xi\right)$ is the concave conjugate function

$$
\rho_{\epsilon}^{*}\left(\frac{1}{2} \xi\right)=\inf _{s>0} \frac{1}{2} \xi s^{2}-\rho_{\epsilon}(s) .
$$

It is shown in [26] that the infimum in 222 is achieved when $\xi=\rho_{\epsilon}^{\prime}(s) / s$. Consequently, for the regularization term $\mathbf{Q}(\mathbf{x})$ in $[15)$, we can write

$$
\mathbf{Q}(\mathbf{x}) \leq R(\mathbf{x}, \boldsymbol{\xi})=\frac{1}{2} \sum_{d \in \Delta} \mathbf{x}^{t} \mathbf{F}_{d}^{t} \boldsymbol{\Omega}_{d} \mathbf{F}_{d} \mathbf{x}-\sum_{d \in \Delta} \sum_{i=1}^{N} \rho_{\epsilon}^{*}\left(\frac{1}{2} \xi_{d}(i)\right)
$$

where $\boldsymbol{\xi}=\left(\boldsymbol{\xi}_{1}, \ldots, \boldsymbol{\xi}_{Q}\right), \boldsymbol{\xi}_{q}=\left(\xi_{q}(1), \ldots, \xi_{q}(N)\right)$ for $q=1, \ldots, Q$, with all its components positive, and $\boldsymbol{\Omega}_{d}$ is a diagonal matrix with entries

$$
\Omega_{d}(i, i)=\xi_{d}(i) .
$$

For a given $\mathbf{x}$, the first inequality in (24) becomes an equality if (see [26] for details),

$$
\xi_{d}^{\mathbf{x}}(i)=\min \left(1 /\left|\omega_{d}^{\mathbf{x}}(i)\right|^{2}, 1 / \epsilon^{2}\right)= \begin{cases}\frac{1}{\left|\omega_{d}^{\mathbf{x}}(i)\right|^{2}} & \text { for }\left|\omega_{d}^{\mathbf{x}}(i)\right| \geq \epsilon \\ \frac{1}{\epsilon^{2}} & \text { for } 0 \leq\left|\omega_{d}^{\mathbf{x}}(i)\right| \leq \epsilon\end{cases}
$$

where $\omega_{d}^{\mathbf{x}}(i)$ is defined from $\mathbf{x}$ in $(12)$. Then we can apply standard MajorizationMinimization (MM) methods [31]. Given $\mathbf{x}^{k}, \mathbf{a}^{k}, \mathbf{s}^{k}$ and defining

$$
L^{k}(\mathbf{x})=\frac{\beta}{2} \sum_{q}\left\|\mathbf{B}_{q}\left(\mathbf{s}_{q}^{k}\right) \mathbf{x}-\mathbf{W} \mathbf{a}_{q}^{k}\right\|^{2}+\sum_{q} \boldsymbol{\lambda}_{q}^{k^{t}}\left(\mathbf{B}_{q}\left(\mathbf{s}_{q}^{k}\right) \mathbf{x}-\mathbf{W} \mathbf{a}_{q}^{k}\right)
$$

it can be easily shown that

$$
L^{k}\left(\mathbf{x}^{k}\right)+\alpha \mathbf{Q}\left(\mathbf{x}^{k}\right) \geq L^{k}\left(\mathbf{x}^{k+1}\right)+\alpha \mathbf{Q}\left(\mathbf{x}^{k+1}\right)
$$

where

$$
\begin{aligned}
\mathbf{x}^{k+1} & =\arg \min _{\mathbf{x}}\left\{\frac{\beta}{2} \sum_{q}\left\|\mathbf{B}_{q}\left(\mathbf{s}_{q}^{k}\right) \mathbf{x}-\mathbf{W} \mathbf{a}_{q}\right\|^{2}+\alpha R\left(\mathbf{x}, \boldsymbol{\xi}^{\mathbf{x}^{k}}\right)\right. \\
& \left.+\sum_{q} \boldsymbol{\lambda}_{q}^{k^{t}}\left(\mathbf{B}_{q}\left(\mathbf{s}_{q}^{k}\right) \mathbf{x}-\mathbf{W} \mathbf{a}_{q}^{k}\right)\right\} .
\end{aligned}
$$


From $(29)$, the optimization step in (18) produces the following linear equation for $\mathbf{x}^{k+1}$

$$
\mathbf{x}^{k+1}=\left[\beta \sum_{q} \mathbf{B}_{q}^{k}{ }_{q}^{t}\left(\mathbf{s}_{q}^{k}\right) \mathbf{B}_{q}^{k}\left(\mathbf{s}_{q}^{k}\right)+\alpha \sum_{d \in \Delta} \mathbf{F}_{d}^{t} \boldsymbol{\Omega}_{d}^{k} \mathbf{F}_{d}\right]^{-1} \sum_{q} \mathbf{B}_{q}^{k}\left(\mathbf{s}_{q}^{k}\right)^{t}\left[\beta \mathbf{W} \mathbf{a}_{q}^{k}-\lambda_{q}^{k}\right]
$$

where

$$
\Omega_{d}^{k}(i, i)=\min \left(1 /\left|\omega_{d}^{\mathbf{x}^{k}}(i)\right|^{2}, 1 / \epsilon^{2}\right) .
$$

The optimization step in 19 for each $\mathbf{a}_{q}$ produces

$$
\begin{aligned}
\mathbf{a}_{q}^{k+1} & =\underset{\mathbf{a}_{q}}{\arg \min }\left\{\frac{\eta}{2}\left\|\mathbf{\Phi} \mathbf{W} \mathbf{a}_{q}-\mathbf{y}_{q}\right\|^{2}+\tau\left\|\mathbf{a}_{q}\right\|_{1}\right. \\
& \left.+\frac{\beta}{2}\left\|\mathbf{B}_{q}^{k}\left(\mathbf{s}_{q}^{k}\right) \mathbf{x}^{k+1}-\mathbf{W} \mathbf{a}_{q}\right\|^{2}-\boldsymbol{\lambda}_{q}^{k^{t}}\left(\mathbf{B}_{q}^{k}\left(\mathbf{s}_{q}^{k}\right) \mathbf{x}-\mathbf{W} \mathbf{a}_{q}\right)\right\}
\end{aligned}
$$

which is equivalent to

$$
\begin{aligned}
\mathbf{a}_{q}^{k+1} & =\arg \min _{\mathbf{a}_{q}}\left\{\frac{\eta}{2}\left\|\mathbf{\Phi} \mathbf{W} \mathbf{a}_{q}-\mathbf{y}_{q}\right\|^{2}\right. \\
& \left.+\frac{\beta}{2}\left\|\mathbf{B}_{q}^{k}\left(\mathbf{s}_{q}^{k}\right) \mathbf{x}^{k+1}-\boldsymbol{\lambda}_{q}^{k}-\mathbf{W} \mathbf{a}_{q}\right\|^{2}+\tau\left\|\mathbf{a}_{q}\right\|_{1}\right\} .
\end{aligned}
$$

The above equation can be rewritten as

$$
\mathbf{a}_{q}^{k+1}=\arg \min _{\mathbf{a}_{q}}\left\|\Phi^{\prime} \mathbf{W} \mathbf{a}_{q}-\mathbf{J}^{\prime}\right\|^{2}+\tau\left\|\mathbf{a}_{q}\right\|_{1},
$$

where

$$
\mathbf{J}^{\prime}=\left[\begin{array}{c}
\sqrt{\frac{\eta}{2}} \mathbf{y}_{q} \\
\sqrt{\frac{\beta}{2}}\left(\mathbf{B}_{q}^{k}\left(\mathbf{s}_{q}^{k}\right) \mathbf{x}^{k+1}-\boldsymbol{\lambda}_{q}^{k}\right)
\end{array}\right] \text { and } \boldsymbol{\Phi}^{\prime}=\left[\begin{array}{c}
\sqrt{\frac{\eta}{2}} \mathbf{\Phi} \\
\sqrt{\frac{\beta}{2}} \mathbf{I}
\end{array}\right]
$$

with $\mathbf{I}$ the $D \times D$ identity matrix.

The above optimization problem can be solved using the algorithm in [32].

To estimate the registration parameters in $(20)$, we have to solve

$$
\mathbf{s}_{q}^{k+1}=\underset{\mathbf{s}_{q}}{\operatorname{argmin}} \frac{\beta}{2}\left\|\mathbf{B}_{q}\left(\mathbf{s}_{q}\right) \mathbf{x}^{k+1}-\mathbf{W} \mathbf{a}_{q}^{k+1}\right\|^{2} .
$$

Notice that we could use regularization of the parameters to be estimated as we did in [17. However, we have experimentally observed that regularization was not needed for this problem. $\mathbf{B}_{q}\left(\mathbf{s}_{q}\right) \mathbf{x}$, can be approximated by expanding 
it into its first-order Taylor series around the previous value $\mathbf{s}_{q}^{k}$. Hence obtaining (see [25, 33])

$$
\begin{aligned}
\mathbf{B}\left(\mathbf{s}_{q}^{k+1}\right) \mathbf{x}^{k+1} & \approx \mathbf{B}\left(\mathbf{s}_{q}^{k}\right) \mathbf{x}^{k+1} \\
& +\left[\mathbf{O}_{q 1}\left(\mathbf{s}_{q}^{k}\right) \mathbf{x}^{k+1}, \mathbf{O}_{q 2}\left(\mathbf{s}_{q}^{k}\right) \mathbf{x}^{k+1}, \mathbf{O}_{q 3}\left(\mathbf{s}_{q}^{k}\right) \mathbf{x}^{k+1}\right]\left(\mathbf{s}_{q}^{k+1}-\mathbf{s}_{q}^{k}\right),
\end{aligned}
$$

where $\mathbf{O}_{q i}\left(\mathbf{s}_{q}^{k}\right) \mathbf{x}^{k+1}=\mathbf{A} \mathbf{H}_{q} \mathbf{N}_{i}\left(\mathbf{s}_{q}^{k}\right) \mathbf{x}^{k+1}$. The values of $\mathbf{N}_{i}\left(\mathbf{s}_{q}^{k}\right) \mathbf{x}^{k+1}$ can be calculated using

$$
\begin{aligned}
& {\left[\mathbf{N}_{1}\left(\mathbf{s}_{q}^{k}\right) \mathbf{x}, \mathbf{N}_{2}\left(\mathbf{s}_{q}^{k}\right) \mathbf{x}, \mathbf{N}_{3}\left(\mathbf{s}_{q}^{k}\right) \mathbf{x}\right]=} \\
& {\left[\left(\mathbf{P}_{1}\left(\mathbf{s}_{q}^{k}\right) \mathbf{M}_{1}\left(\mathbf{s}_{q}^{k}\right)+\mathbf{P}_{2}\left(\mathbf{s}_{q}^{k}\right) \mathbf{M}_{2}\left(\mathbf{s}_{q}^{k}\right), \mathbf{M}_{1}\left(\mathbf{s}_{q}^{k}\right), \mathbf{M}_{2}\left(\mathbf{s}_{q}^{k}\right)\right],\right.}
\end{aligned}
$$

where

$$
\begin{aligned}
\mathbf{M}_{1}\left(\mathbf{s}_{q}^{k}\right) & =\left(\mathbf{I}-\mathbf{D}_{\mathbf{b}_{q}\left(\mathbf{s}_{q}\right)}\right)\left(\mathbf{L}_{\mathbf{t r}\left(s_{q}\right)}-\mathbf{L}_{\mathbf{t l}\left(s_{q}\right)}\right)+\mathbf{D}_{\mathbf{b}_{q}\left(\mathbf{s}_{q}\right)}\left(\mathbf{L}_{\mathbf{b r}\left(s_{q}\right)}-\mathbf{L}_{\mathbf{b l}\left(s_{q}\right)}\right) \\
\mathbf{M}_{2}\left(\mathbf{s}_{q}^{k}\right) & =\left(\mathbf{I}-\mathbf{D}_{\mathbf{a}_{q}\left(\mathbf{s}_{q}\right)}\right)\left(\mathbf{L}_{\mathbf{b l}\left(s_{q}\right)}-\mathbf{L}_{\mathbf{t l}\left(s_{q}\right)}\right)+\mathbf{D}_{\mathbf{a}_{q}\left(\mathbf{s}_{q}\right)}\left(\mathbf{L}_{\mathbf{b r}\left(s_{q}\right)}-\mathbf{L}_{\mathbf{t r}\left(s_{q}\right)}\right) \\
\mathbf{P}_{1}\left(\mathbf{s}_{q}^{k}\right) & =-\left[\mathbf{D}_{\mathbf{u}} \sin \left(\theta_{q}^{k}\right)+\mathbf{D}_{\mathbf{v}} \cos \left(\theta_{q}^{k}\right)\right] \\
\mathbf{P}_{2}\left(\mathbf{s}_{q}^{k}\right) & =\left[\mathbf{D}_{\mathbf{u}} \cos \left(\theta_{q}^{k}\right)-\mathbf{D}_{\mathbf{v}} \sin \left(\theta_{q}^{k}\right),\right.
\end{aligned}
$$

and $\mathbf{D}_{\mathbf{u}}$ and $\mathbf{D}_{\mathbf{v}}$ are diagonal matrices whose diagonals are the vectors $\mathbf{u}$ and $\mathbf{v}$, respectively. Substituting (37) into (36), we obtain the final update equation as follows

$$
\mathbf{s}_{q}^{k+1}=\left[\boldsymbol{\Lambda}_{q}^{k}\right]^{-1}\left(\boldsymbol{\Upsilon}_{q}^{k}+\boldsymbol{\Lambda}_{q}^{k} \mathbf{s}_{q}^{k}\right)=\mathbf{s}_{q}^{k}+\left[\boldsymbol{\Lambda}_{q}^{k}\right]^{-1} \mathbf{\Upsilon}_{q}^{k},
$$

where $\boldsymbol{\Lambda}_{q}^{k}$ and $\boldsymbol{\Upsilon}_{q}^{k}$ correspond to the $q$-th observation at the $k$-th iteration. The $i, j \in\{1,2,3\}$ elements of these matrices are given by

$$
\begin{aligned}
\Lambda_{q i j}^{k} & =\left[\mathbf{A} \mathbf{H}_{q} \mathbf{N}_{i}\left(\mathbf{s}_{q}^{k}\right) \mathbf{x}^{k+1}\right]^{t} \mathbf{A} \mathbf{H}_{q} \mathbf{N}_{j}\left(\mathbf{s}_{q}^{k}\right) \mathbf{x}^{k+1}, \\
\Upsilon_{q i}^{k} & =\left[\mathbf{A H}_{q} \mathbf{N}_{i}\left(\mathbf{s}_{q}^{k}\right) \mathbf{x}^{k+1}\right]^{t}\left(\mathbf{W a}_{q}^{k+1}-\mathbf{A} \mathbf{H}_{q} \mathbf{N}_{i}\left(\mathbf{s}_{q}^{k}\right) \mathbf{x}^{k+1}\right) .
\end{aligned}
$$

The complete CSSR algorithm is presented in Algorithm 1

\section{Experimental Results}

To evaluate the proposed algorithm, experiments were carried out on two types of images: simulated compressed LR images, and real LR images which are synthetically compressed using a measurement matrix $\boldsymbol{\Phi}$. In both cases, $\boldsymbol{\Phi}$ is constructed using a circulant Toeplitz matrix with entries drawn from a Bernoulli distribution. We utilized a 3-level Haar wavelet transform as the transform basis. Using exhaustive search, the values of the parameters utilized were $\tau=1.25 \times 10^{-2}, \alpha=5 \times 10^{-4}$, for all experiments. For the $\mathrm{SNR}=40 \mathrm{~dB}$ case, $\eta=49.29, \beta=3.85 \times 10^{-5}$, and for the $\mathrm{SNR}=30 \mathrm{~dB}$ case, $\eta=4.93, \beta=$ $3.85 \times 10^{-4}$. 


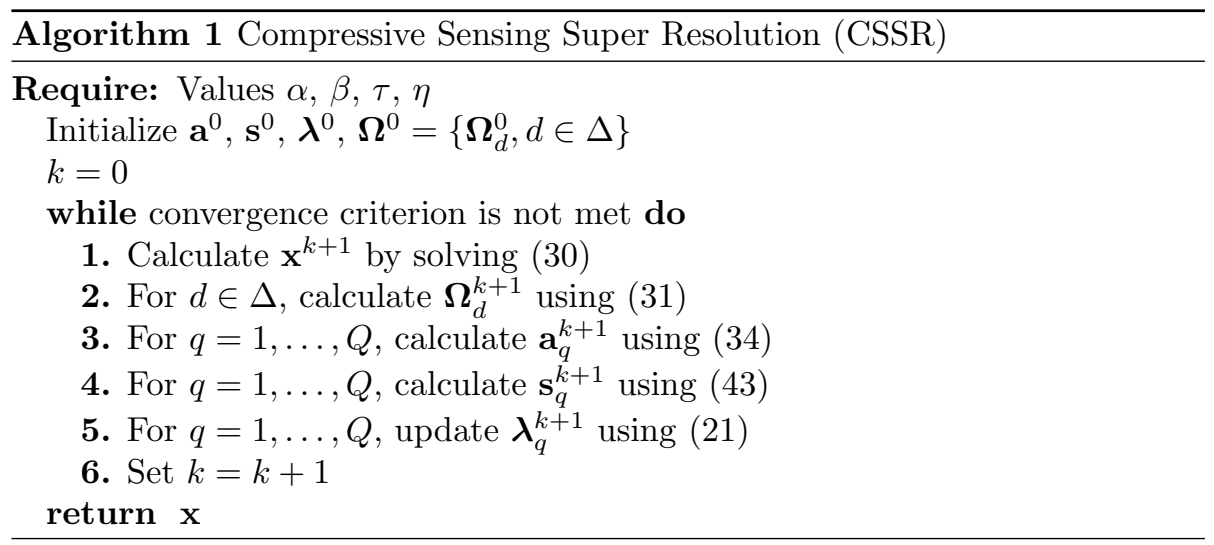

The algorithm stops when either a maximum number of iterations (60 in all experiments) is reached, or when

$$
\frac{\operatorname{norm}\left(\mathbf{x}^{k}-\mathbf{x}^{k-1}\right)}{\operatorname{norm}\left(\mathbf{x}^{k-1}\right)} \leq 10^{-4} .
$$

To estimate the initial value of the registration parameters $\mathbf{s}_{q}^{0}$, in 43 , the following minimization problem, similar to (36), was solved

$$
\mathbf{s}_{q}^{0}=\underset{\mathbf{s}_{q}}{\operatorname{argmin}}\left\|\mathbf{C}\left(\mathbf{s}_{q}\right) \mathbf{W} \mathbf{a}_{R}^{0}-\mathbf{W} \mathbf{a}_{q}^{0}\right\|^{2}, \quad q=2, \ldots, Q
$$

The obtained registration parameters are then upsampled to the HR level. The subscript $R$ denotes the reference image which corresponds to the first reconstructed LR observation.

\subsection{Simulated Images}

To generate all simulated LR images from an original image we use the following procedure. The original HR image is first randomly rotated and horizontally and vertically displaced. Then it is blurred with a Gaussian blur, with known variance. Next it is down-sampled by a variable zooming factor P. It is compressed using $\boldsymbol{\Phi}$, with variable compression ratio R, and finally white Gaussian noise is added to the CS observations with variable signal-to-noise ratios (SNR). $Q$ different observations are generated using this procedure. An example of the degradation process, on the Cameraman image, is shown in Figure 2.

As performance measure we use the Peak Signal to Noise Ratio (PSNR) calculated using

$$
P S N R=10 \log \frac{N \max \left(\mathbf{x}_{\text {orig }}\right)^{2}}{\left\|\mathbf{x}_{\text {est }}-\mathbf{x}_{\text {orig }}\right\|^{2}},
$$

where $\mathbf{x}_{\text {orig }}$ and $\mathbf{x}_{\text {est }}$ are $N \times 1$ vectors, representing the original and the estimated HR image, respectively, and $\max \left(\mathbf{x}_{\text {orig }}\right)$ is the maximum possible value of the image $\mathbf{x}_{\text {orig }}$ (the value is equal to 255 for an 8-bit image). 


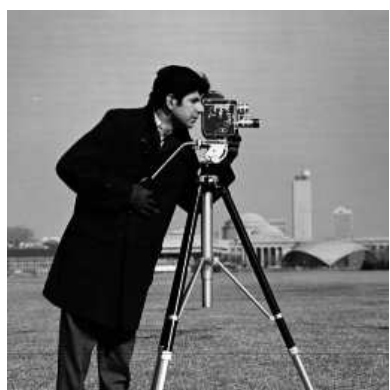

(a)

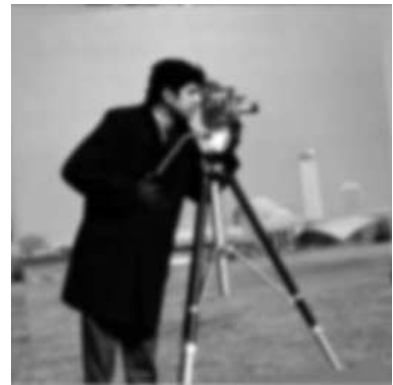

(c)

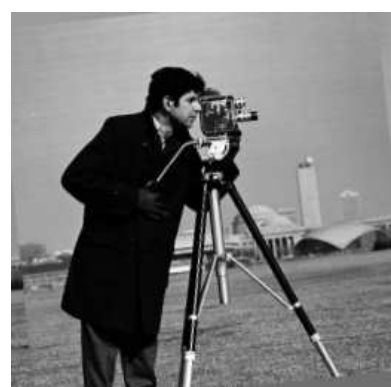

(b)

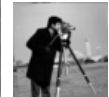

(d)

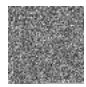

(e)

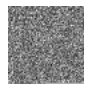

(f)

Figure 2: Degradation procedure. (a) Original image, (b) Warped image, (c) Blurred image, Var $=3$, (d) Down-sampled image, $\mathrm{P}=4$, (e) Compressed acquisition, $\mathrm{R}=0.8$, (f) Noise added, $\mathrm{SNR}=40 \mathrm{~dB}$

\subsubsection{Compressed Sensing Reconstruction}

This experiment concentrates on the CS reconstruction process, to study the performance of the CSSR algorithm. We use two simulated LR images as follows. With the HR Shepp-Logan image of size $256 \times 256$ pixels, shown in Figure 3 (a), we used a blur with variance $=3$, zooming factor $\mathrm{P}=2$, and warping matrix corresponding respectively to the following rotation and displacement vectors: $[0,0,0]^{t},[-0.1047,2,-3]^{t}$. The two simulated LR images are shown in Figure $3(\mathrm{~b}, \mathrm{c})$. These simulated LR images are then compressed with compression ratio $\mathrm{R}=0.8$, and noise is added with $\mathrm{SNR}=30 \mathrm{~dB}$.

Let us examine the first iteration resulting LR estimations using (33), without the registration regularization term. The estimated images are shown in Figure 3(d,e), and the PSNR values of the reconstructed LR images are 40.22 $\mathrm{dB}$ and $40.16 \mathrm{~dB}$, respectively. As the CSSR process advances, the inclusion of the registration term allows for a better extraction of the information in the compressed observations; the final estimated LR images are shown in Figure 3(f,g), with PSNR values being $42.88 \mathrm{~dB}$ and $42.77 \mathrm{~dB}$, respectively.

For the Cameraman image shown in Figure 4(a), the downsampled images are shown in Figure 4 (b,c), the initially estimated LR images are shown in Figure 


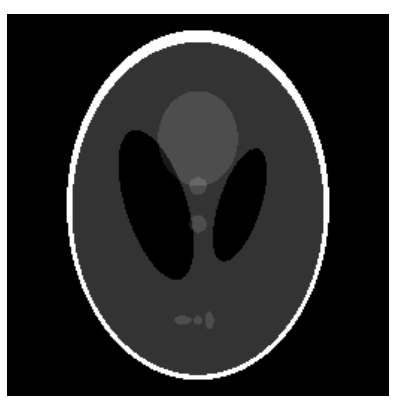

(a)

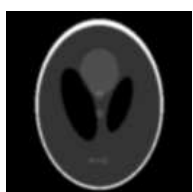

(b)

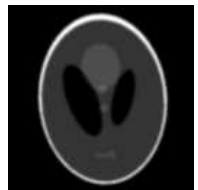

(c)

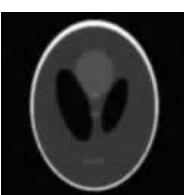

(d)

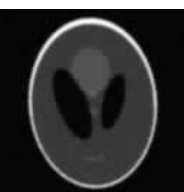

(e)

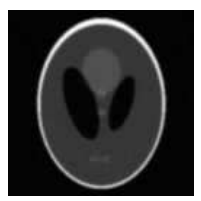

(f)

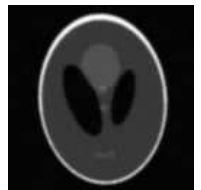

(g)

Figure 3: Reconstruction of LR images. (a) Original HR Shepp-Logan image, $(\mathrm{b}, \mathrm{c})$ Downsampled images $Q=2, \mathrm{P}=2$, Blur Var $=3, \mathrm{R}=0.8, \mathrm{SNR}=30 \mathrm{~dB},(\mathrm{~d}, \mathrm{e})$ First estimates of the LR images, PSNR $=40.22 \mathrm{~dB}$ and , $40.16 \mathrm{~dB}$ respectively, (f,g) Final estimates of the LR images, PSNR $=42.88 \mathrm{~dB}$ and $42.77 \mathrm{~dB}$ respectively.

4(d,e), with PSNR=35.52 dB and $35.29 \mathrm{~dB}$, respectively. The final estimated LR images are shown in Figure $4(\mathrm{f}, \mathrm{g})$, with PSNRs equal to $35.55 \mathrm{~dB}$ and 35.34 $\mathrm{dB}$, respectively.

For the Lena image shown in Figure $5(\mathrm{a})$, the downsampled images are shown in Figure 5(b,c), the initially estimated LR images are shown in Figure 5 (d,e), with PSNR $=32.45 \mathrm{~dB}$ and $32.28 \mathrm{~dB}$, respectively. The final estimated LR images are shown in Figure 5(f,g), with PSNRs equal to $34.24 \mathrm{~dB}$ and 34.07 $\mathrm{dB}$, respectively.

Notice that the inclusion of the registration regularization term in 33 greatly contributes to the PSNR improvement when using the CSSR algorithm. This improvement will be very useful when super resolving the LR observations to estimate the HR image.

\subsubsection{Registration Parameter Estimation}

Two experiments were carried out to establish the CSSR accuracy when estimating the registration parameters. The $256 \times 256$ pixel HR Shepp-Logan image, with $Q=4$, Blur variance $=3, \mathrm{SNR}=40 \mathrm{~dB}$, and $\mathrm{R}=0.8$ was used. $\mathrm{P}=2$ and 4 , were chosen to conduct these two experiments. The real and estimated registration parameters are shown in Table 1, which also contains the absolute errors. Notice that for the first observation, which is the reference observation, the motion vector is $[0,0,0]^{t}$.

\subsubsection{Super Resolution Reconstruction}

This experiment compares the performance of the proposed CSSR algorithm with a set of existing SR algorithms, namely Bicubic Interpolation (BIC), SR using an $l_{1}$ prior [34] (L1S), SR using sparse and non-sparse priors [25] (SnS), a 


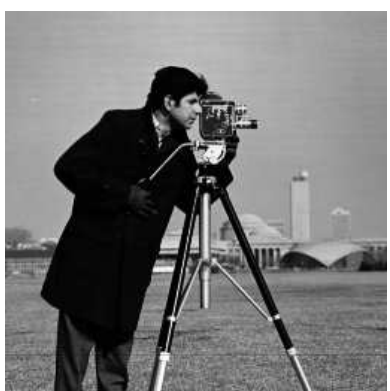

(a)

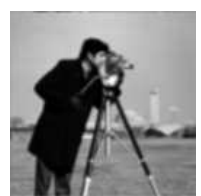

(b)

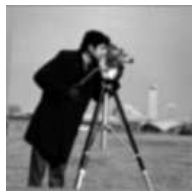

(c)

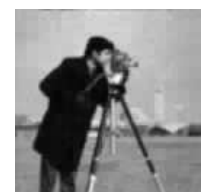

(d)

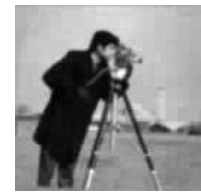

(e)

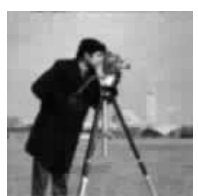

(f)

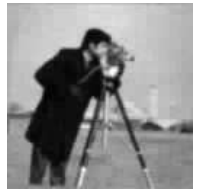

(g)

Figure 4: Reconstruction of LR images. (a) Cameraman original HR image, (b,c) Downsampled images $Q=2, \mathrm{P}=2$, Blur Var $=3, \mathrm{R}=0.8, \mathrm{SNR}=30 \mathrm{~dB},(\mathrm{~d}, \mathrm{e})$ First estimates of the LR images, PSNR $=35.52 \mathrm{~dB}$ and $35.29 \mathrm{~dB}$, respectively, $(\mathrm{f}, \mathrm{g})$ Final estimates of the LR images, PSNR $=35.55 \mathrm{~dB}$ and $35.34 \mathrm{~dB}$, respectively.

fast and robust SR [35] (FRSR), and a robust SR method [36] (RSR). Algorithms L1S 34 and $\mathrm{SnS}$ 25] estimate all their parameters. Since algorithms FRSR [35] and RSR [36] need some parameters to be set, we performed an exhaustive search to find the parameters resulting in the maximum PSNR values. We used the Cameraman, Shepp-Logan and Lena images. The number of observations was $Q=4$. For our CSSR, we used a unity compression ratio $\mathrm{R}=1.0$, to compare with those SR algorithms which do not use CS observations. Notice that we still use CS but with $100 \%$ of the measurements which is equivalent to using $\mathbf{\Phi}=\mathbf{I}$. Every experiment was carried out three times and the tabulated measures are the average values of the three experiments. The results are tabulated in Table 2 .

Figure 6 compares the performance of SR algorithms as a function of the variance of the blur affecting the image. Using $Q=4, \mathrm{SNR}=40 \mathrm{~dB}$ and $\mathrm{P}=4$, the PSNR of the CSSR and the investigated SR algorithms, are compared for the Cameraman, Lena, and Shepp-logan images. Notice the relative improvement of the CSSR algorithm, when compared with SR algorithms that do not use compressed sensing.

\subsubsection{The General Case}

In this experiment the overall behavior of the CSSR algorithm is investigated; the compression ratios and zooming factors will be varied and the sequential and alternate approaches compared.

A comparison between the sequential approach and the CSSR algorithm on the Cameraman and Shepp-Logan images is shown in tables 3 and 4 respectively. Figure 7 shows for $\mathrm{P}=2$ and 4 with $Q=4$, Blur variance $3, \mathrm{SNR}=40 \mathrm{~dB}$, 


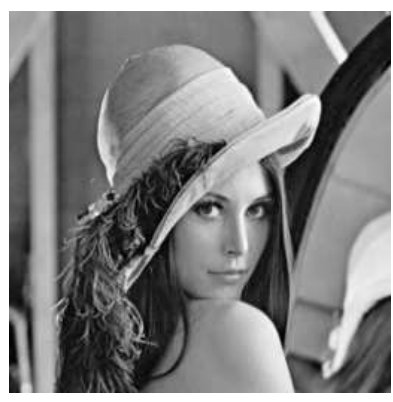

(a)

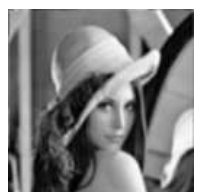

(b)

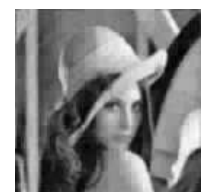

(d)

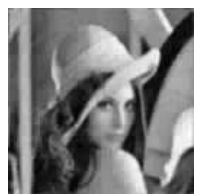

(f)

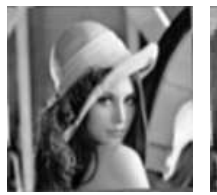

(c)

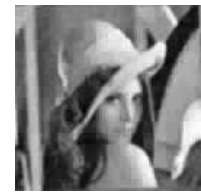

(e)

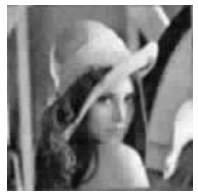

(g)

Figure 5: Reconstruction of LR images. (a) Lena original HR image, (b,c) Downsampled images $Q=2, \mathrm{P}=2$, Blur Var $=3, \mathrm{R}=0.8, \mathrm{SNR}=30 \mathrm{~dB}$, (d,e) First estimates of the LR images, PSNR $=40.22 \mathrm{~dB}$ and $40.16 \mathrm{~dB}$, respectively, (f,g) Final estimates of the LR images, PSNR $=42.88 \mathrm{~dB}$ and $42.77 \mathrm{~dB}$, respectively.

$\mathrm{R}=0.8$, the estimated Shepp-Logan images.

The obtained results show how the alternate approach outperform the sequential. It is important to pause to examine the obtained results. For a $256 \times 256 \mathrm{HR}$ image, the total number of pixels is 65536 . If we use a zooming factor $\mathrm{P}=4$, then the size of the LR image will be $64 \times 64$, and the total number of pixels 4096. Using a compression ratio $\mathrm{R}=0.8$, the number of projections is 3277. Instead of saving 65536 pixel values, the CSSR algorithm uses only 3277 values, a percentage of $4 \times 5.0 \%$, still adequate to obtain a good HR image, see Figure 7(b).

Let us now examine the behavior of the CSSR algorithm as a function of several factors. For $Q=4, \mathrm{P}=4, \mathrm{SNR}=30 \mathrm{~dB}$, Figure 8 shows the behavior of CSSR when the compression ratio and blur vary.

Shown in Figure 9, for both images using a variable $\mathrm{R}, Q=4$, Blur variance $5, \mathrm{SNR}=30 \mathrm{~dB}$, for two values of $\mathrm{P}=2$ and 4 , when changing the zooming factor, the performance degrades with increasing P. However, this difference in performance is less noticeable than with the super resolution algorithms it is compared against. This becomes clear in Table 2, where the dB differences for the CSSR algorithm, on the average, is less than $1 \mathrm{~dB}$, while it has big differences for the tested SR algorithms. For example, for the Shepp-Logan image, the average difference is around $7 \mathrm{~dB}$ for blur variances 5 and 9, for the L1S algorithm, while the differences for the CSSR algorithm were all less than $1 \mathrm{~dB}$. The performance of the CSSR algorithm does not decrease much when increasing the zooming factor and the blur variance affecting the CS observations.

Figure 10 shows the results obtained for both images, with $Q=4$, Blur Var $=5$, $\mathrm{P}=2$. In general, and as expected, better PSNRs were obtained with less noise and less compression. Notice, however, the interesting behavior of the test on 


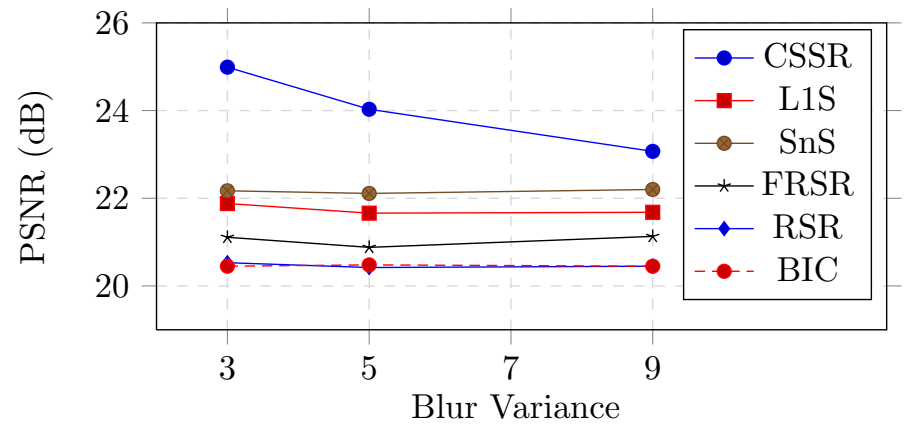

(a)

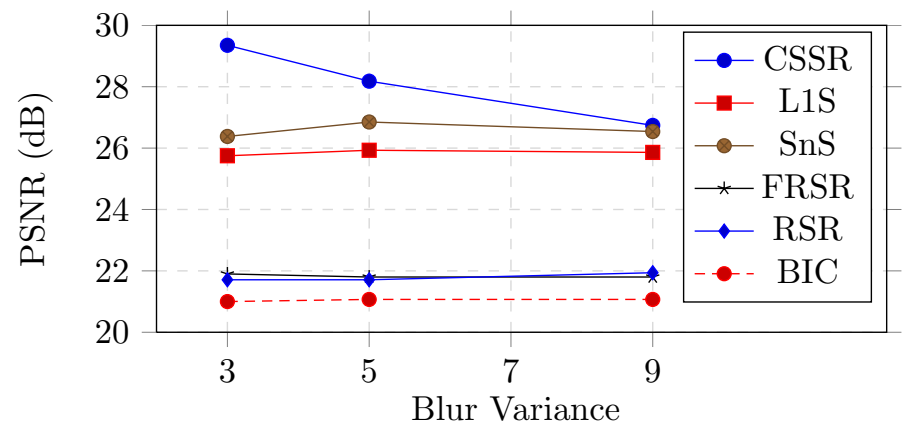

(b)

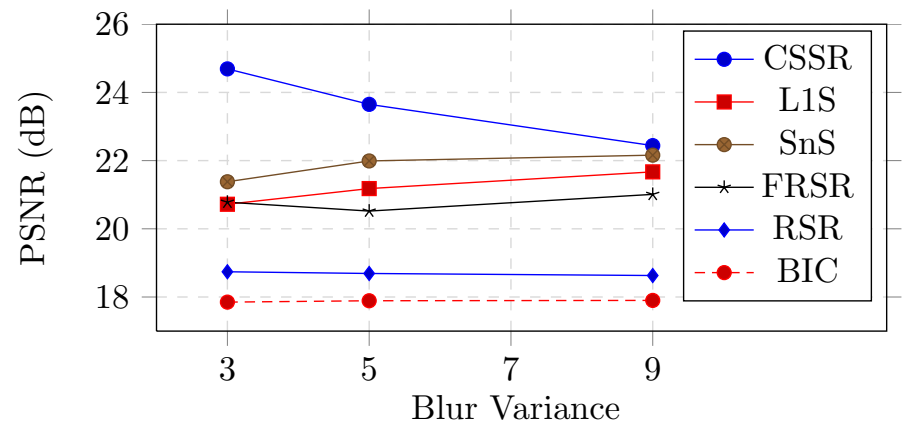

(c)

Figure 6: Comparison among SR algorithms and the CSSR algorithm. $\mathrm{P}=4$, $\mathrm{SNR}=40 \mathrm{~dB}, Q=4$, and for CSR, $\mathrm{R}=1.0$. (a) Cameraman image, (b) Lena, (c) Shepp-Logan image. 
Table 1: Registration parameter estimation of CSSR for the Shepp-Logan image, $Q=4$, Blur var $=3, \mathrm{SNR}=40 \mathrm{~dB}, \mathrm{R}=0.8$.

\begin{tabular}{|c|c|c|c|c|}
\hline \multirow{2}{*}{$\begin{array}{c}\text { Zooming } \\
\text { Factor }\end{array}$} & \multicolumn{4}{|c|}{ Observation, $q=2$} \\
\hline & Motion Vector & $\theta_{q}$ & $a_{q}$ & $b_{q}$ \\
\hline 2,4 & True & .05236 & 2.00 & -3.00 \\
\hline \multirow{2}{*}{2} & Estimated & .05229 & 1.999 & -3.002 \\
\hline & Abs. Error & $7.0 \mathrm{e}-5$ & $1.0 \mathrm{e}-3$ & $2.0 \mathrm{e}-3$ \\
\hline \multirow{2}{*}{4} & Estimated & .05163 & 2.033 & -3.057 \\
\hline & Abs. Error & $7.3 \mathrm{e}-4$ & $3.3 \mathrm{e}-2$ & $5.7 \mathrm{e}-2$ \\
\hline & \multicolumn{4}{|c|}{ Observation, $\mathrm{q}=3$} \\
\hline 2,4 & True & -.06981 & -1.0 & -2.0 \\
\hline \multirow{2}{*}{2} & Estimated & -0.06916 & -1.002 & -2.005 \\
\hline & Abs. Error & $6.5 \mathrm{e}-4$ & $2.0 \mathrm{e}-3$ & $5 \mathrm{e}-3$ \\
\hline \multirow{2}{*}{4} & Estimated & -.06902 & -1.012 & -2.046 \\
\hline & Abs. Error & $7.9 \mathrm{e}-4$ & $1.2 \mathrm{e}-2$ & $4.6 \mathrm{e}-2$ \\
\hline & \multicolumn{4}{|c|}{ Observation, $q=4$} \\
\hline 2,4 & True & -.03491 & 3.0 & -1.0 \\
\hline \multirow{2}{*}{2} & Estimated & -0.03478 & 3.001 & -1.001 \\
\hline & Abs. Error & $1.3 \mathrm{e}-4$ & $1.0 \mathrm{e}-3$ & $1.0 \mathrm{e}-3$ \\
\hline \multirow{2}{*}{4} & Estimated & -.03351 & 2.999 & -1.041 \\
\hline & Abs. Error & $1.4 \mathrm{e}-3$ & $1.0 \mathrm{e}-3$ & $4.1 \mathrm{e}-2$ \\
\hline
\end{tabular}

the Shepp-Logan image where better quality restored images are obtained from noisier observations for low compression ratios. This is very likely due to the very flat nature of this particular image.

\subsection{Real Images}

This experiment uses real LR observations, the underlying HR image being unavailable. The experiments were carried out to visually assess the estimated HR image, and to study the effect of the number of input images $Q$, on the estimated HR image.

In the first experiment we used noisy LR images of a car, the first four of which are shown in Figure 11(a). The estimated high resolution image, using bilinear interpolation of one of the LR reconstructed images, is shown in Figure 11(b). The estimated HR images using the CSSR algorithm are shown in Figures 11(c, d), for number of input images $Q=4$ and 16 , respectively. We used a compression ratio $\mathrm{R}=0.8$, and a zooming factor, $\mathrm{P}=2$. The better performance of the proposed CSSR in comparison to bilinear interpolation is clearly observable. Notice also that a better performance is achieved by increasing the number of input images; this becomes apparent when trying to read the letters written on the windscreen of the car; they are more readable in Figure 11(d) than that in Figure 11(c). Notice also the artifacts on the left hand side of the estimated image, due to warping and Fourier implementation of the blur convolution. 


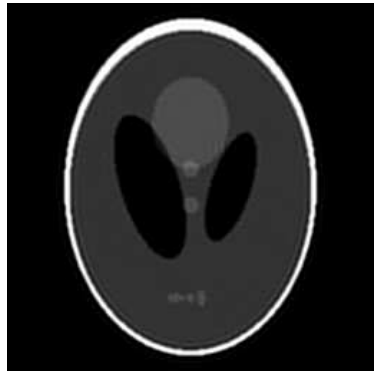

(a)

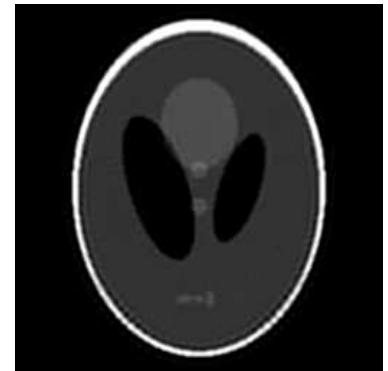

(b)

Figure 7: Image super resolution from simulated images, $Q=4$, Blur Var $=3$, $\mathrm{SNR}=40 \mathrm{~dB}$. (a) Estimated image using $\mathrm{P}=2, \mathrm{R}=0.8$, (b) Estimated image using $\mathrm{P}=4, \mathrm{R}=0.8$

In the second experiment we used noisy LR images of a toy, the first four of which are shown in Figure 12(a). The bilinear interpolation of one reconstructed LR image is shown in Figure 12(b). The estimated images using the CSSR algorithm, are shown in Figures 12 (c,d), for number of input images $Q=4$ and 16 , respectively. The compression ratio was equal to 0.8 , and the zooming factor was equal to 2 . Similarly, the best result was obtained using more input images (as shown in Figure 12(d)), when compared to both the interpolated image in Figure 12(b), and to the CSSR estimated image in Figure 12(c). The estimated image in Figure 12(d) is smoother with sharper edges and better quality.

\subsection{Real Passive Millimeter Wave Images}

In this experiment we used four noisy real $100 \times 30$ PMMW LR observations of a man hiding a threat attached to his left arm, as shown in Figure 13(a). The unregistered images were synthetically compressed using a compression ratio $\mathrm{R}=0.8$. In the first part we used a zooming factor $\mathrm{P}=2$, and compare the estimated image using CSSR, shown in Figure 13(c) with the image shown in Figure 13.(b), obtained by bilinear interpolation of the reconstructed reference image. In the estimated image using CSSR, the discontinuity in the arm, which refers to the threat location, appears more prominent than that in the interpolated image. This becomes even clearer in the next experiment, using a zooming factor $\mathrm{P}=4$, under the same conditions. The estimated image using CSSR, shown in Figure 13(e), is again compared with the interpolated image, shown in Figure 13(d). In addition, the edges of the legs look sharper. Notice that the estimated image in the latter case has achieved a factor of increase in resolution of 16, a good result taking into account the low quality of the compressed input images. This is a very important preprocessing step which should help improve the performance of any threat detection algorithm. 

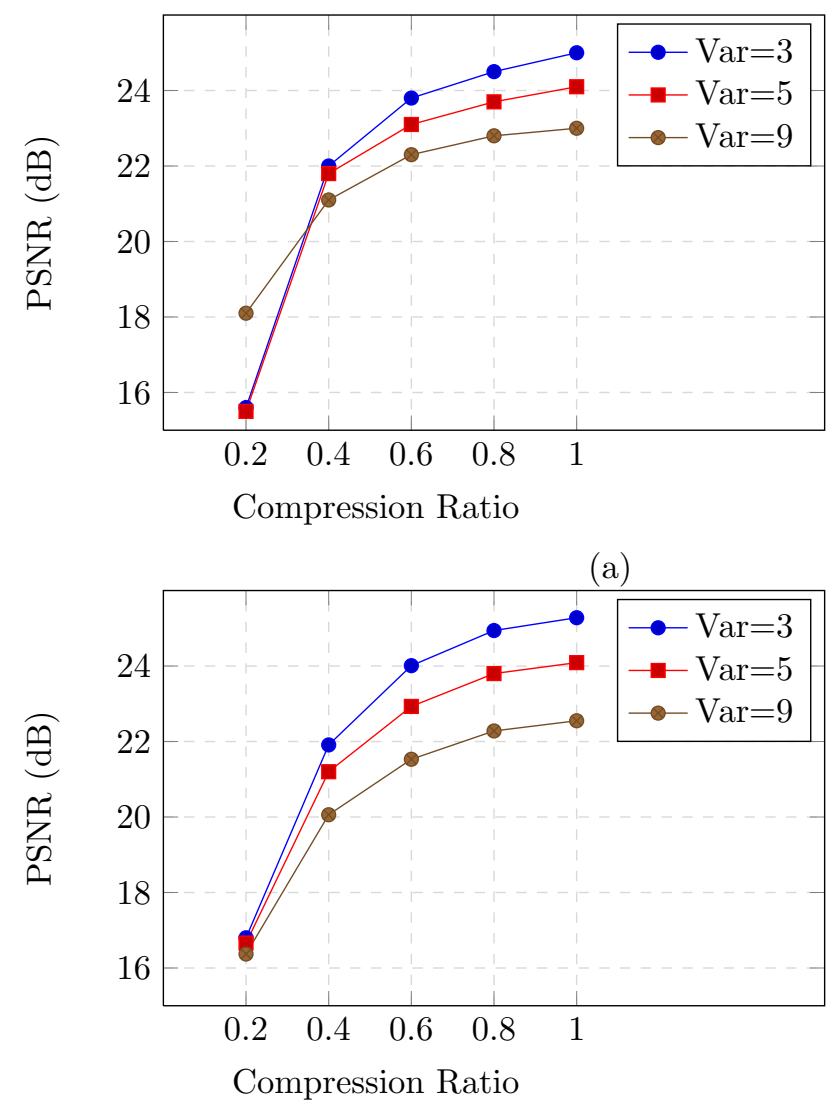

(b)

Figure 8: Comparison for performance measure of proposed CSSR vs blur variance. $Q=4, \mathrm{P}=4, \mathrm{SNR}=30 \mathrm{~dB}$. on (a) Cameraman, (b) Shepp-Logan.

\section{Conclusions}

In this work we have proposed a method to obtain an HR image from a set of LR CS observations. The method combines CS and LR to HR reconstruction using ADMM and base the HR robust reconstruction on an efficient registration procedure and a new sparsity promoting prior. We have experimentally shown that this simultaneous reconstruction outperforms the method, that first performs LR reconstruction to then obtain an HR image from a set of LR observations. The experiments carried out show a very good performance in comparison with existing HR methods which do not use CS observations. We have also shown how the proposed method can be used to improve the quality of PMMW images to be used in detection tasks. 


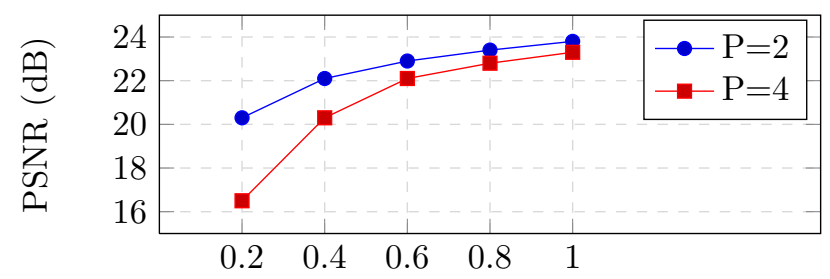

Compression Ratio

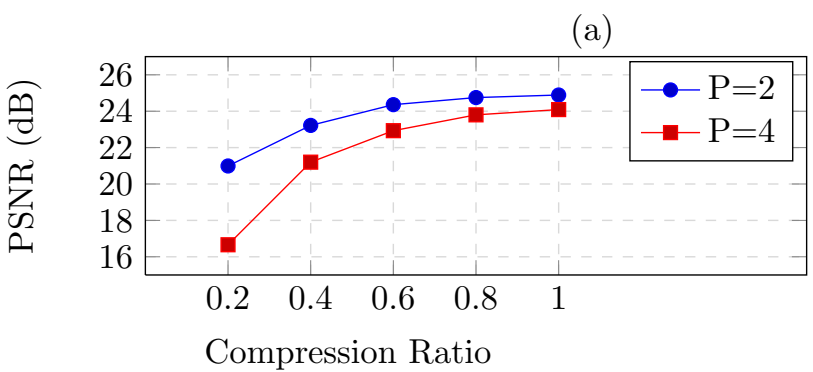

(b)

Figure 9: Performance of the proposed CSSR for various zooming factors and compression ratios. $Q=4$, Blur Var $=5, \mathrm{SNR}=30 \mathrm{~dB}$. (a) Cameraman image, (b) Shepp-Logan image.

\section{Acknowledgement}

This paper has been supported by The European Union, Erasmus Mundus program, the Spanish Ministry of Economy and Competitiveness under project TIN2013-43880-R, the European Regional Development Fund (FEDER), the U.S. Department of Energy (DE-NA0002520), and a Northwestern University Catalyst grant.

\section{References}

[1] E. Candes, M. Wakin, An introduction to compressive sampling, Signal Processing Magazine, IEEE 25 (2) (2008) 21-30.

[2] M. Fornasier, H. Rauhut, Compressive sensing, in: Handbook of mathematical methods in imaging, Springer, 2011, pp. 187-228.

[3] E. Candes, J. Romberg, Practical signal recovery from random projections., Wavelet Applications in Signal and Image Processing XI, Proc. SPIE Conf. 5914.

[4] M. Duarte, M. Davenport, D. Takhar, J. Laska, T. Sun, K. Kelly, R. Baraniuk, Single-pixel imaging via compressive sampling, Signal Processing Magazine, IEEE 25 (2) (2008) 83-91. doi:10.1109/MSP.2007.914730. 

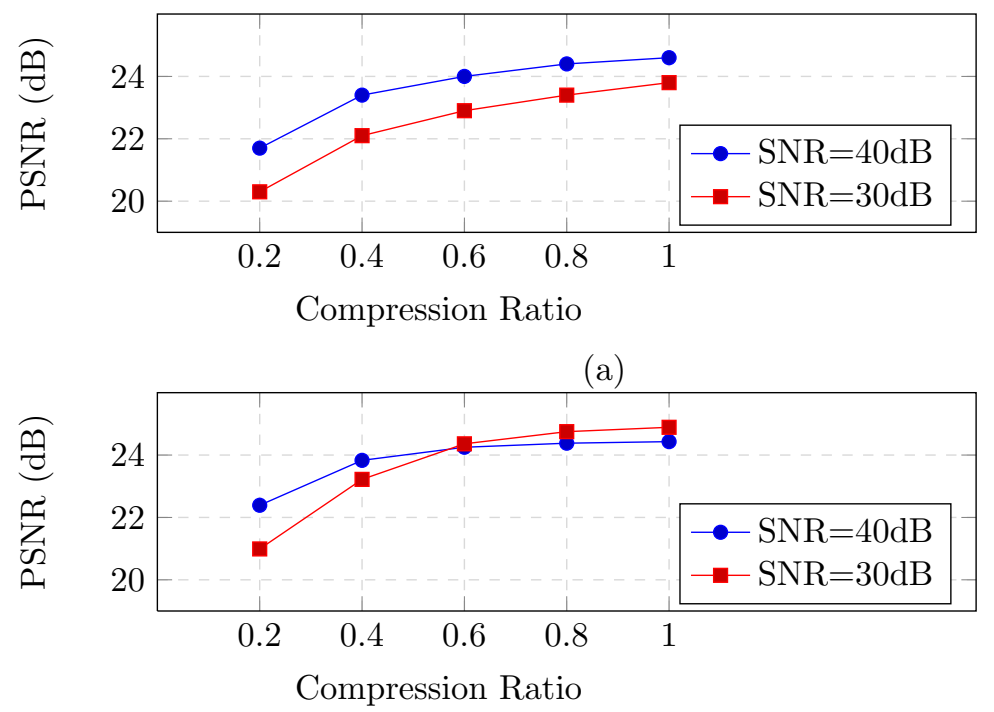

(b)

Figure 10: Performance of the proposed CSSR for various signal to noise ratios, of the additive noise, and compression ratios. $Q=4, \mathrm{P}=2$, Blur Var=5, $\mathrm{SNR}=30 \mathrm{~dB}$. (a) Cameraman image, (b) Shepp-Logan image.

[5] W. L. Chan, K. Charan, D. Takhar, K. F. Kelly, R. Baraniuk, D. Mittleman, A single-pixel terahertz imaging system based on compressed sensing, Applied Physics Letters 93 (12) (2008) 121105-121105-3. doi: $10.1063 / 1.2989126$

[6] A. Heidari, D. Saeedkia, A 2d camera design with a single-pixel detector, in: Infrared, Millimeter, and Terahertz Waves, 2009. IRMMW-THz 2009. 34th International Conference on, 2009, pp. 1-2. doi:10.1109/ICIMW. 2009.5324725

[7] T. Li, X. Wang, W. Wang, A. K. Katsaggelos, Compressive video sensing with limited measurements, Journal of Electronic Imaging 22 (4) (2013) 043003-043003. doi:10.1117/1.JEI.22.4.043003.

URL http://dx.doi.org/10.1117/1.JEI.22.4.043003

[8] A. Sankaranarayanan, C. Studer, R. Baraniuk, Cs-muvi: Video compressive sensing for spatial-multiplexing cameras, in: Computational Photography (ICCP), 2012 IEEE International Conference on, 2012, pp. 1-10. doi: 10.1109/ICCPhot.2012.6215212.

[9] Y. C. Eldar, G. Kutyniok, Compressed sensing: theory and applications, Cambridge University Press, 2012. 


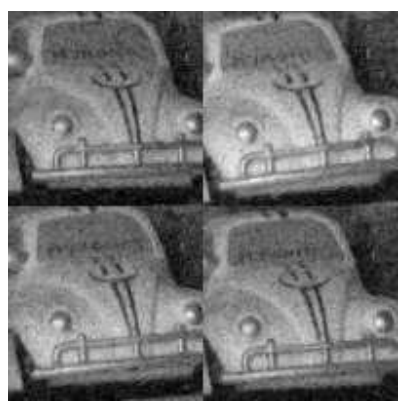

(a)

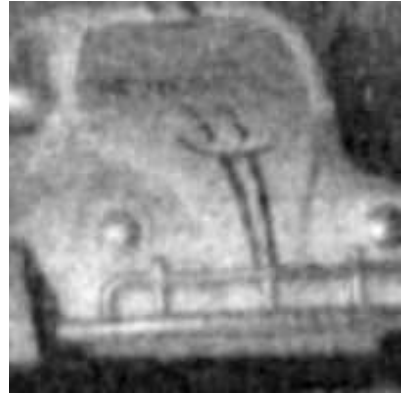

(b)

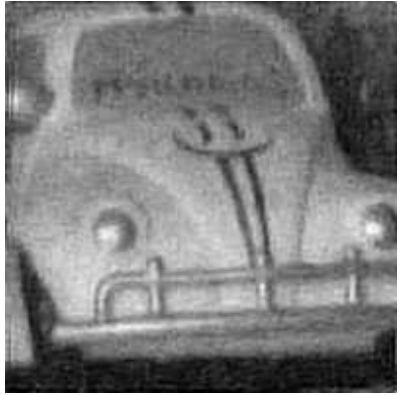

(c)

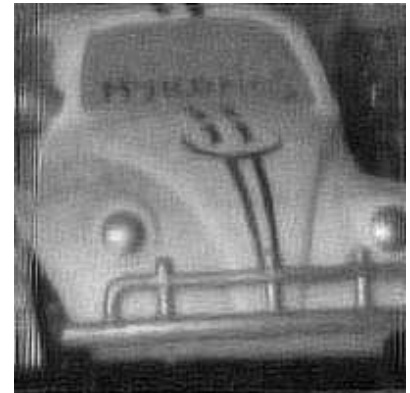

(d)

Figure 11: Image super resolution from real observations, $\mathrm{R}=0.8, \mathrm{P}=2$. (a) First 4 LR images (b) Bilinear interpolation of one reconstructed LR image, (c) Estimated HR image using the CSSR algorithm, with $Q=4$, (d) Estimated HR image using the CSSR algorithm, with $Q=16$.

[10] H. Babcock, J. Moffitt, Y. Cao, X. Zhuang, Fast compressed sensing analysis for superresolution imaging using l1-homotopy, Optics Express 21 (23) (2013) 28583-28596. doi:10.1364/OE.21.028583.

[11] L. Spinoulas, B. Amizic, M. Vega, R. Molina, A. Katsaggelos, Simultaneous bayesian compressive sensing and blind deconvolution, in: Signal Processing Conference (EUSIPCO), 2012 Proceedings of the 20th European, 2012, pp. $1414-1418$.

[12] B. Amizic, L. Spinoulas, R. Molina, A. Katsaggelos, Compressive blind image deconvolution, Image Processing, IEEE Transactions on 22 (10) (2013) 3994-4006. doi:10.1109/TIP.2013.2266100.

[13] C. He, L. Liu, L. Xu, M. Liu, M. Liao, Learning based compressed sensing for sar image super-resolution, Selected Topics in Applied Earth Observations and Remote Sensing, IEEE Journal of 5 (4) (2012) 1272-1281. 


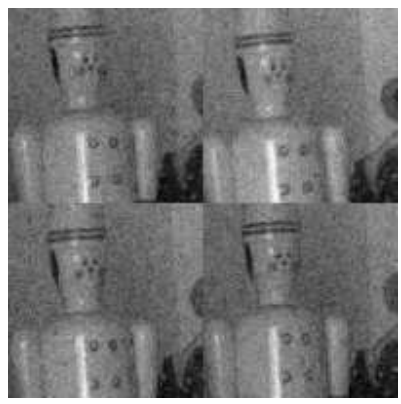

(a)

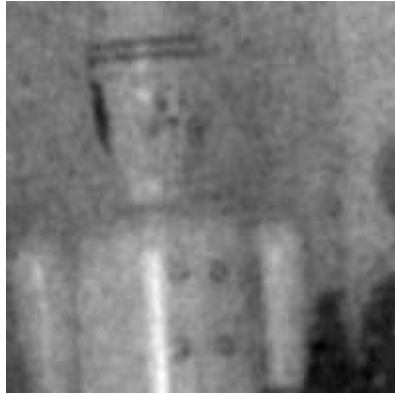

(b)

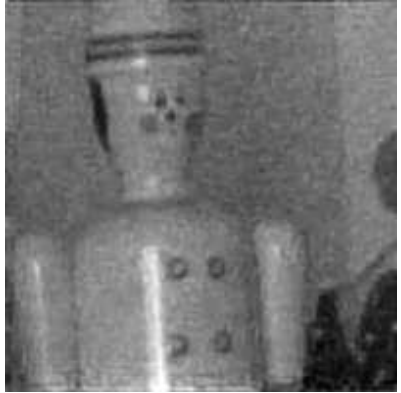

(c)

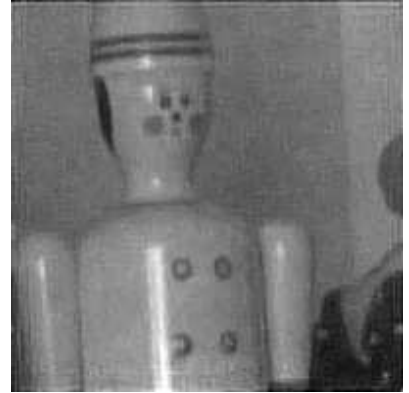

(d)

Figure 12: Image super resolution from real observations. $\mathrm{R}=0.8, \mathrm{P}=2$. (a) First 4 LR images, (b) Bilinear interpolation of one reconstructed LR image, (c) Estimated HR image using the CSSR algorithm, with $Q=4$, (d) Estimated HR image using the CSSR algorithm, with $Q=16$.

[14] S. Yang, F. Sun, M. Wang, Z. Liu, L. Jiao, Novel super resolution restoration of remote sensing images based on compressive sensing and example patches-aided dictionary learning, in: Multi-Platform/Multi-Sensor Remote Sensing and Mapping (M2RSM), 2011 International Workshop on, IEEE, 2011, pp. 1-6.

[15] P. Sen, S. Darabi, Compressive image super-resolution, in: Signals, Systems and Computers, 2009 Conference Record of the Forty-Third Asilomar Conference on, 2009, pp. 1235-1242. doi:10.1109/ACSSC.2009.5469968

[16] W. Saafin, M. Vega, R. Molina, A. K. Katsaggelos, Image super-resolution from compressed sensing observations, in: Image Processing (ICIP), 2015 IEEE International Conference on Image Processing, 2015.

[17] W. Saafin, S. Villena, M. Vega, R. Molina, A. Katsaggelos, Pmmw image super resolution from compressed sensing observations, in: European Signal Processing Conference (EUSIPCO), 2015. 


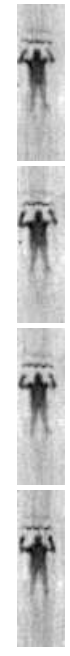

(a)

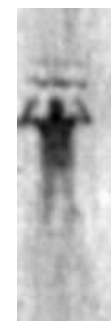

(b)

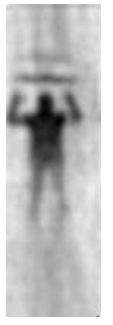

(c)

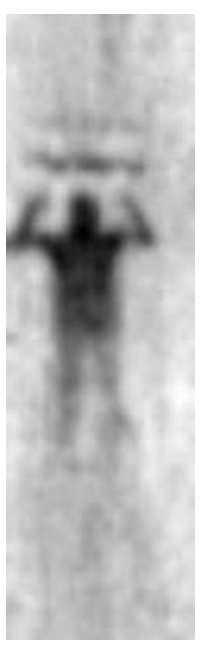

(d)

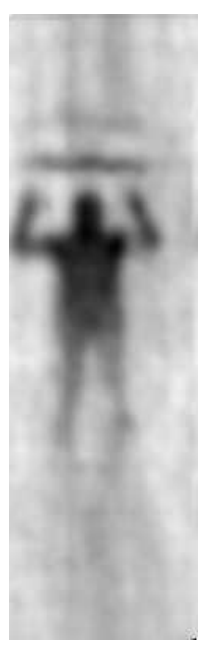

(e)

Figure 13: PMMW image super resolution from multiple observations, $Q=4$, $\mathrm{R}=0.8$. (a) $100 \times 30 \mathrm{LR}$ images, (b) Bilinear interpolation from one reconstructed image, $\mathrm{P}=2$, (c) Estimated HR image using the CSSR algorithm, $\mathrm{P}=2$, (d) Bilinear interpolation from one reconstructed image, $\mathrm{P}=4$, (e) Estimated $\mathrm{HR}$ image using the CSSR algorithm, $\mathrm{P}=4$.

[18] J. Yang, J. Wang, L. Li, A new algorithm for passive millimeter-wave image enhancement, in: Signal Processing Systems (ICSPS), 2010 2nd International Conference on, Vol. 3, IEEE, 2010, pp. V3-507.

[19] Y. Li, Y. Li, J. Chen, Y. Hou, Passive millimeter-wave image restoration based on improved algorithm of nonlinear extrapolation in frequency space, International Journal of Digital Content Technology and its Applications 5 (5) (2011) 42-49.

[20] P. Chen, T. Zou, J. Chen, Z. Gao, J. Xiong, The application of improved pso algorithm in pmmw image ostu threshold segmentation, in: Applied Mechanics and Materials, Vol. 721, Trans Tech Publ, 2015, pp. 779-782.

[21] H. Chen, S. Lee, R. Rao, M. Slamani, P. Varshney, Imaging for concealed weapon detection: a tutorial overview of development in imaging sensors and processing, Signal Processing Magazine, IEEE 22 (2) (2005) 52-61.

[22] H. Lee, D. Lee, S. Yeom, J. Son, V. Guschin, S. Kim, Passive millimeter wave imaging and analysis for concealed object detection, in: Data Mining 
and Intelligent Information Technology Applications (ICMiA), 2011 3rd International Conference on, IEEE, 2011, pp. 98-101.

[23] N. Gopalsami, S. Liao, T. W. Elmer, E. R. Koehl, A. Heifetz, A. C. Raptis, L. Spinoulas, A. K. Katsaggelos, Passive millimeter-wave imaging with compressive sensing, OPTICAL ENGINEERING 51 (9). doi: $\{10.1117 / 1.0 \mathrm{E} .51 .9 .091614\}$.

[24] N. Gopalsami, T. Elmer, S. Liao, R. Ahern, A. Heifetz, A. Raptis, M. Luessi, D. Babacan, A. Katsaggelos, Compressive sampling in passive millimeter-wave imaging, in: SPIE Defense, Security, and Sensing, International Society for Optics and Photonics, 2011, pp. 80220I-80220I.

[25] S. Villena, M. Vega, S. Babacan, R. Molina, A. Katsaggelos, Bayesian combination of sparse and non-sparse priors in image super resolution, Digital Signal Processing 23 (2) (2013) 530-541.

[26] S. D. Babacan, R. Molina, M. N. Do, A. K. Katsaggelos, Bayesian blind deconvolution with general sparse image priors, in: Computer Vision-ECCV 2012, Springer, 2012, pp. 341-355.

[27] A. K. Katsaggelos, R. Molina, J. Mateos, Super resolution of images and video, Synthesis Lectures on Image, Video, and Multimedia Processing 1 (1) (2007) 1-134.

[28] S. Boyd, N. Parikh, E. Chu, B. Peleato, J. Eckstein, Distributed optimization and statistical learning via the alternating direction method of multipliers, Foundations and Trends® in Machine Learning 3 (1) (2011) $1-122$.

[29] Y.-H. Xiao, Z.-F. Jin, An alternating direction method for linearconstrained matrix nuclear norm minimization, Numerical Linear Algebra with Applications 19 (3) (2012) 541-554.

[30] R. T. Rockafellar, Convex analysis, no. 28, Princeton university press, 1997.

[31] K. Lange, Optimization, Springer-Verlag, 2013.

[32] S.-J. Kim, K. Koh, M. Lustig, S. Boyd, D. Gorinevsky, An interior-point method for large-scale 11-regularized least squares, Selected Topics in Signal Processing, IEEE Journal of 1 (4) (2007) 606-617. doi:10.1109/JSTSP. 2007.910971

[33] Y. He, K. Yap, L. Chen, L.-P. Chau, A nonlinear least square technique for simultaneous image registration and super-resolution, Image Processing, IEEE Transactions on 16 (11) (2007) 2830-2841.

[34] S. Villena, M. Vega, R. Molina, A. K. Katsaggelos, Bayesian superresolution image reconstruction using an 11 prior, in: Image and Signal Processing and Analysis, 2009. ISPA 2009. Proceedings of 6th International Symposium on, IEEE, 2009, pp. 152-157. 
[35] S. Farsiu, M. Robinson, M. Elad, P. Milanfar, Fast and robust multiframe super resolution, IEEE Transactions on Image Processing 13 (10) (2004) $1327-1344$.

[36] A. Zomet, A. Rav-Acha, S. Peleg, Robust super-resolution, in: Computer Vision and Pattern Recognition, 2001. CVPR 2001. Proceedings of the 2001 IEEE Computer Society Conference on, Vol. 1, 2001, pp. I-645-I-650 vol.1. doi:10.1109/CVPR.2001.990535. 
Table 2: Comparison of state-of-the-art SR algorithms with the CSSR algorith$\mathrm{m}$, with $Q=4$ and for CSSR $\mathrm{R}=1.0$. Every experiment was repeated three times and the shown values are the average values. In bold are the highest PSNR values.

\begin{tabular}{|c|c|c|c|c|c|c|c|}
\hline \multirow{3}{*}{\multicolumn{2}{|c|}{$\begin{array}{c}\text { Image } \\
\text { Blur Var } \\
\text { SNR }\end{array}$}} & \multicolumn{6}{|c|}{ Cameraman } \\
\hline & & \multicolumn{2}{|c|}{3} & \multicolumn{2}{|c|}{5} & \multicolumn{2}{|c|}{9} \\
\hline & & 40 & 30 & 40 & 30 & 40 & 30 \\
\hline ( Alg & $\overline{\mathrm{P}}$ & \multicolumn{6}{|c|}{ Average PSNR, dB } \\
\hline \multirow{2}{*}{ BIC } & 2 & 22.5 & $\overline{\overline{22.7}}$ & 222.4 & 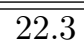 & 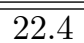 & $\overline{22.4}$ \\
\hline & 4 & 20.5 & 20.4 & 20.5 & 20.5 & 20.5 & 20.4 \\
\hline \multirow{2}{*}{ L1S } & 2 & 24.1 & 24.5 & 23.9 & 24.2 & 24.1 & 23.9 \\
\hline & 4 & 21.9 & 22.1 & 21.7 & 21.3 & 21.7 & 21.6 \\
\hline \multirow{2}{*}{$\mathrm{SnS}$} & 2 & 24.1 & 23.7 & 24.1 & 23.4 & 24.2 & 23.3 \\
\hline & 4 & 22.2 & 22.4 & 22.1 & 22.0 & 22.2 & 22.1 \\
\hline \multirow{2}{*}{ FRSR } & 2 & 20.9 & 21.5 & 19.3 & 19.8 & 21.5 & 20.6 \\
\hline & 4 & 21.1 & 22.3 & 20.9 & 21.2 & 21.1 & 21.8 \\
\hline \multirow{2}{*}{ RSR } & 2 & 22.5 & 22.2 & 21.1 & 21.6 & 21.3 & 22.0 \\
\hline & 4 & 20.5 & 20.6 & 20.4 & 20.5 & 20.5 & 20.6 \\
\hline \multirow{2}{*}{ CSSR } & 2 & 25.9 & 24.8 & 24.6 & 23.8 & 23.4 & 22.6 \\
\hline & 4 & 25.0 & 24.1 & 24.0 & 23.3 & 23.1 & 22.3 \\
\hline \multicolumn{2}{|c|}{ Image } & \multicolumn{6}{|c|}{ Lena } \\
\hline \multirow{2}{*}{$\mathrm{BIC}$} & 2 & 24.7 & 24.7 & 24.6 & 24.6 & 24.5 & 24.4 \\
\hline & 4 & 21.1 & 21.0 & 21.1 & 21.0 & 21.1 & 21.1 \\
\hline \multirow{2}{*}{ L1S } & 2 & 27.0 & 27.4 & 27.2 & 27.5 & 26.7 & 27.4 \\
\hline & 4 & 25.8 & 25.7 & 25.9 & 25.4 & 25.9 & 26.0 \\
\hline \multirow{2}{*}{$\mathrm{SnS}$} & 2 & 27.7 & 27.5 & 28.4 & 27.6 & 28.3 & 27.5 \\
\hline & 4 & 26.4 & 26.3 & 26.9 & 25.9 & 26.5 & 26.4 \\
\hline \multirow{2}{*}{ FRSR } & 2 & 20.5 & 20.8 & 21.2 & 20.9 & 22.0 & 20.1 \\
\hline & 4 & 21.9 & 22.4 & 21.8 & 23.2 & 21.8 & 24.9 \\
\hline \multirow{2}{*}{ RSR } & 2 & 23.1 & 25.2 & 25.0 & 25.1 & 24.2 & 23.0 \\
\hline & 4 & 21.7 & 21.9 & 21.7 & 21.8 & 21.9 & 22.0 \\
\hline \multirow{2}{*}{ CSSR } & 2 & 31.1 & 29.0 & 29.4 & 27.6 & 27.3 & 26.0 \\
\hline & 4 & 29.4 & 27.4 & 28.2 & 26.4 & 26.7 & 25.2 \\
\hline \multicolumn{2}{|c|}{ Image } & \multicolumn{6}{|c|}{ Shepp-Logan } \\
\hline \multirow{2}{*}{$\mathrm{BIC}$} & 2 & 20.5 & 20.5 & 20.5 & 20.8 & 20.3 & 20.3 \\
\hline & 4 & 17.9 & 17.8 & 17.9 & 17.9 & 17.9 & 17.9 \\
\hline \multirow{2}{*}{ L1S } & 2 & 25.7 & 27.9 & 27.9 & 30.3 & 26.2 & 27.7 \\
\hline & 4 & 20.7 & 20.4 & 21.2 & 21.1 & 21.7 & 21.6 \\
\hline $\mathrm{SnS}$ & 2 & 24.1 & 23.3 & 24.1 & 23.2 & 24.1 & 23.2 \\
\hline & 4 & 21.4 & 21.8 & 22.0 & 22.1 & 22.2 & 21.5 \\
\hline & 2 & 20.0 & 20.8 & 20.2 & 21.9 & 20.2 & 21.0 \\
\hline FRSR & 4 & 20.8 & 20.5 & 20.5 & 20.9 & 21.0 & 20.8 \\
\hline & 2 & 21.1 & 21.0 & 21.4 & 21.0 & 21.3 & 21.1 \\
\hline KSR & 4 & 18.7 & 18.8 & 18.7 & 18.7 & 18.6 & 18.4 \\
\hline & 2 & 25.8 & 26.4 & 24.4 & 24.9 & 22.9 & 23.2 \\
\hline CSSR & 4 & 24.7 & 25.3 & 2 Z3.7 & 24.1 & 22.4 & 22.6 \\
\hline
\end{tabular}


Table 3: Comparison of the sequential approach and the CSSR algorithm for the Cameraman image, using $Q=4$. Every experiment was repeated three times and the shown values are the average values. In bold are the highest PSNR values.

\begin{tabular}{|c|c|c|c|c|c|c|c|c|}
\hline \multirow{2}{*}{\multicolumn{3}{|c|}{$\begin{array}{c}\text { Blur Var } \\
\text { SNR }\end{array}$}} & \multicolumn{2}{|c|}{3} & \multicolumn{2}{|c|}{5} & \multicolumn{2}{|c|}{9} \\
\hline & & & 40 & 30 & 40 & 30 & 40 & 30 \\
\hline $\mathrm{P}$ & Alg & $\mathrm{R}$ & \multicolumn{6}{|c|}{ Average PSNR, dB } \\
\hline \multirow{10}{*}{2} & 7 & 0.2 & 19.4 & 19.9 & 18.9 & 19.6 & 17.5 & 18.9 \\
\hline & $\stackrel{\widetilde{a}}{\vec{\rightleftarrows}}$ & 0.4 & 22.2 & 22.1 & 21.7 & 21.6 & 20.7 & 20.8 \\
\hline & $\stackrel{\overrightarrow{0}}{己}$ & 0.6 & 23.8 & 22.9 & 23.0 & 22.2 & 21.9 & 21.3 \\
\hline & ت્ّ & 0.8 & 24.5 & 23.2 & 23.5 & 22.5 & 22.4 & 21.5 \\
\hline & & 1.0 & 24.8 & 23.4 & 23.8 & 22.6 & 22.6 & 21.6 \\
\hline & & 0.2 & 22.2 & 20.7 & 21.7 & 20.3 & 20.8 & 19.8 \\
\hline & $\tau_{1}$ & 0.4 & 24.2 & 22.7 & 23.4 & 22.1 & 22.4 & 21.2 \\
\hline & En & 0.6 & 25.1 & 23.8 & 24.0 & 22.9 & 22.9 & 22.0 \\
\hline & & 0.8 & 25.7 & 24.4 & 24.4 & 23.4 & 23.2 & 22.4 \\
\hline & & 1.0 & 25.9 & 24.8 & 24.6 & 23.8 & 23.4 & 22.6 \\
\hline \multirow{10}{*}{4} & $\bar{\sigma}$ & 0.2 & 15.5 & 13.9 & 15.5 & 14.0 & 14.7 & 13.9 \\
\hline & ت्ञ류 & 0.4 & 18.9 & 15.3 & 18.8 & 15.4 & 18.3 & 15.3 \\
\hline & $\stackrel{\Xi}{\Xi}$ & 0.6 & 21.7 & 15.9 & 21.3 & 15.9 & 20.5 & 15.8 \\
\hline & ت & 0.8 & 23.2 & 15.9 & 22.6 & 16.0 & 21.7 & 16.1 \\
\hline & & 1.0 & 23.9 & 15.9 & 23.1 & 15.9 & 22.1 & 16.0 \\
\hline & & 0.2 & 15.8 & 16.6 & 15.5 & 16.5 & 18.1 & 16.3 \\
\hline & ז & 0.4 & 22.0 & 20.6 & 21.8 & 20.3 & 21.1 & 19.8 \\
\hline & की & 0.6 & 23.8 & 22.5 & 23.1 & 22.1 & 22.3 & 21.2 \\
\hline & & 0.8 & 24.5 & 23.5 & 23.7 & 22.8 & 22.8 & 22.0 \\
\hline & & 1.0 & 25.0 & 24.1 & 24.1 & 23.3 & 23.0 & 22.3 \\
\hline
\end{tabular}


Table 4: Performance of sequential approach, with CSSR algorithm, for the Shepp-Logan image, using $Q=4$. Every experiment was repeated three times and the shown values are the average values. In bold are the highest PSNR values.

\begin{tabular}{|c|c|c|c|c|c|c|c|c|}
\hline \multirow{2}{*}{\multicolumn{3}{|c|}{$\begin{array}{c}\text { Blur Var } \\
\text { SNR }\end{array}$}} & \multicolumn{2}{|c|}{3} & \multicolumn{2}{|c|}{5} & \multicolumn{2}{|c|}{9} \\
\hline & & & 40 & 30 & 40 & 30 & 40 & 30 \\
\hline $\mathrm{P}$ & Alg & $\mathrm{R}$ & \multicolumn{6}{|c|}{ Average PSNR, dB } \\
\hline \multirow{10}{*}{2} & 7 & 0.2 & 17.1 & 18.6 & 16.4 & 18.2 & 15.2 & 17.6 \\
\hline & $\stackrel{\widetilde{a}}{\vec{\rightleftarrows}}$ & 0.4 & 22.8 & 23.3 & 21.9 & 22.2 & 20.3 & 20.9 \\
\hline & $\stackrel{\overrightarrow{0}}{己}$ & 0.6 & 24.3 & 23.9 & 23.1 & 22.8 & 21.5 & 21.5 \\
\hline & ت્ّ & 0.8 & 24.7 & 24.1 & 23.5 & 23.0 & 21.9 & 21.7 \\
\hline & & 1.0 & 24.9 & 24.1 & 23.7 & 23.0 & 22.1 & 21.7 \\
\hline & & 0.2 & 23.3 & 21.7 & 22.4 & 21.0 & 20.7 & 20.0 \\
\hline & $\tau_{1}$ & 0.4 & 25.0 & 24.3 & 23.8 & 23.2 & 22.2 & 21.7 \\
\hline & En & 0.6 & 25.5 & 25.7 & 24.3 & 24.4 & 22.7 & 22.6 \\
\hline & & 0.8 & 25.7 & 26.2 & 24.4 & 24.8 & 22.8 & 23.1 \\
\hline & & 1.0 & 25.8 & 26.4 & 24.4 & 24.9 & 22.9 & 23.3 \\
\hline \multirow{10}{*}{4} & $\bar{\sigma}$ & 0.2 & 14.0 & 15.6 & 14.2 & 15.4 & 13.5 & 15.2 \\
\hline & ت्ञ류 & 0.4 & 19.0 & 20.2 & 18.5 & 19.4 & 17.4 & 18.4 \\
\hline & $\stackrel{\Xi}{\Xi}$ & 0.6 & 22.6 & 22.3 & 21.6 & 21.5 & 20.3 & 20.4 \\
\hline & ت & 0.8 & 23.6 & 22.6 & 22.5 & 21.8 & 21.2 & 20.7 \\
\hline & & 1.0 & 23.9 & 22.7 & 22.8 & 21.9 & 21.5 & 20.8 \\
\hline & & 0.2 & 15.6 & 16.8 & 16.0 & 16.7 & 15.6 & 16.4 \\
\hline & 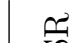 & 0.4 & 23.0 & 21.9 & 22.1 & 21.2 & 20.8 & 20.1 \\
\hline & on & 0.6 & 24.3 & 24.0 & 23.3 & 22.9 & 21.9 & 21.5 \\
\hline & U & 0.8 & 24.6 & 24.9 & 23.5 & 23.8 & 22.2 & 22.3 \\
\hline & & 1.0 & 24.7 & 25.3 & 23.7 & 24.1 & 22.3 & 22.6 \\
\hline
\end{tabular}

